\title{
UCRL-TR-220340
}

LAW RENCE LIVERMORE N A T IO N A L LABORATORY

Analysis of the January 2006 Pepper-Pot Experiments

G. Westenskow, F. Chambers, F. Bieniosek, E. Henestroza

April 3, 2006 
This document was prepared as an account of work sponsored by an agency of the United States Government. Neither the United States Government nor the University of California nor any of their employees, makes any warranty, express or implied, or assumes any legal liability or responsibility for the accuracy, completeness, or usefulness of any information, apparatus, product, or process disclosed, or represents that its use would not infringe privately owned rights. Reference herein to any specific commercial product, process, or service by trade name, trademark, manufacturer, or otherwise, does not necessarily constitute or imply its endorsement, recommendation, or favoring by the United States Government or the University of California. The views and opinions of authors expressed herein do not necessarily state or reflect those of the United States Government or the University of California, and shall not be used for advertising or product endorsement purposes.

This work was performed under the auspices of the U.S. Department of Energy by University of California, Lawrence Livermore National Laboratory under Contract W-7405-Eng-48. 
March 22, 2006

\section{Analysis of the January 2006 Pepper-Pot Experiments ${ }^{1}$}

by Glen Westenskow, Frank Chambers, Frank Bieniosek, Enrique Henestroza

Between January 9-12, 2006 a series of experiments were performed on the DARHT-II injector to measure the beam's emittance. Part of these experiments were pepper-pot measurements. This note describes the analysis of the data, and our conclusions from the experiments.

The experiments were done just after the BCUZ section (see Figure 1). Figure 2 shows the layout near the pepper-pot hardware. We used a beam energy of $2.6 \mathrm{MeV}$ in the calculations based on diode voltage measurements. Subsequent energy spectrometer measurements indicated a beam energy of $2.45 \mathrm{MeV}$. This discrepancy (8\%) impacts directly the numbers reported below for the normalized emittance.

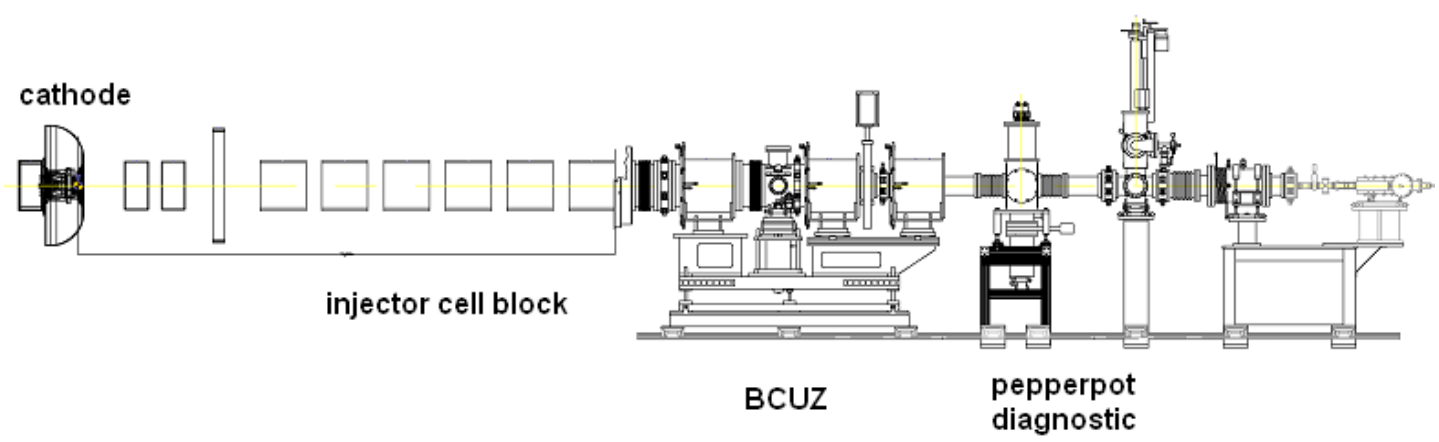

Figure 1. Beam line layout for the pepper-pot measurements.

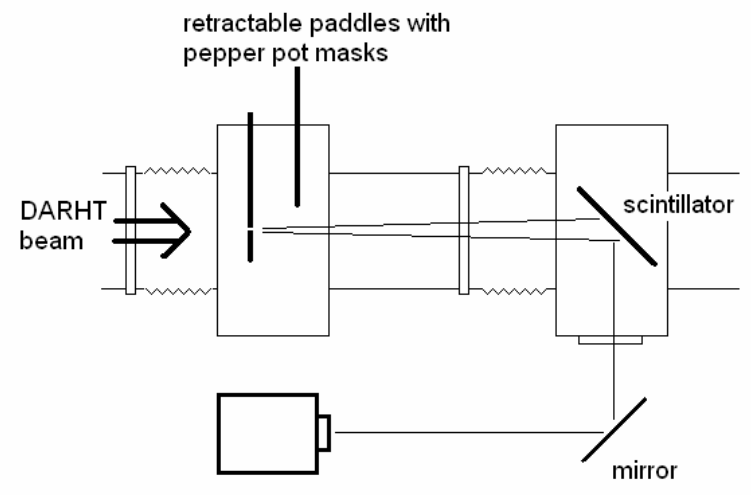

Figure 2. Hardware layout near the pepper pot.

1 . This work has been performed under the auspices of the US DOE by UC-LBNL under contract DEAC03-76SF00098 and by UC-LLNL under contract W-7405-ENG-48. 
There were two square masks used in the experiment. One had $9 \times 9$ holes. The second mask had 13 x 13 holes. All the data in this report is from experiments with the second mask. The separation between holes was $5 \mathrm{~mm}$. The diameter of the holes was $1 \mathrm{~mm}$. Notice there are locations were holes were not drilled in the mask near the bottom left corner (See Figure 4). The scintillator for the pepper-pot images was $90 \mathrm{~cm}$ downstream of the mask. The scintillator was Bicron BC-422. It's light output decay time is $1.6 \mathrm{~ns}$.
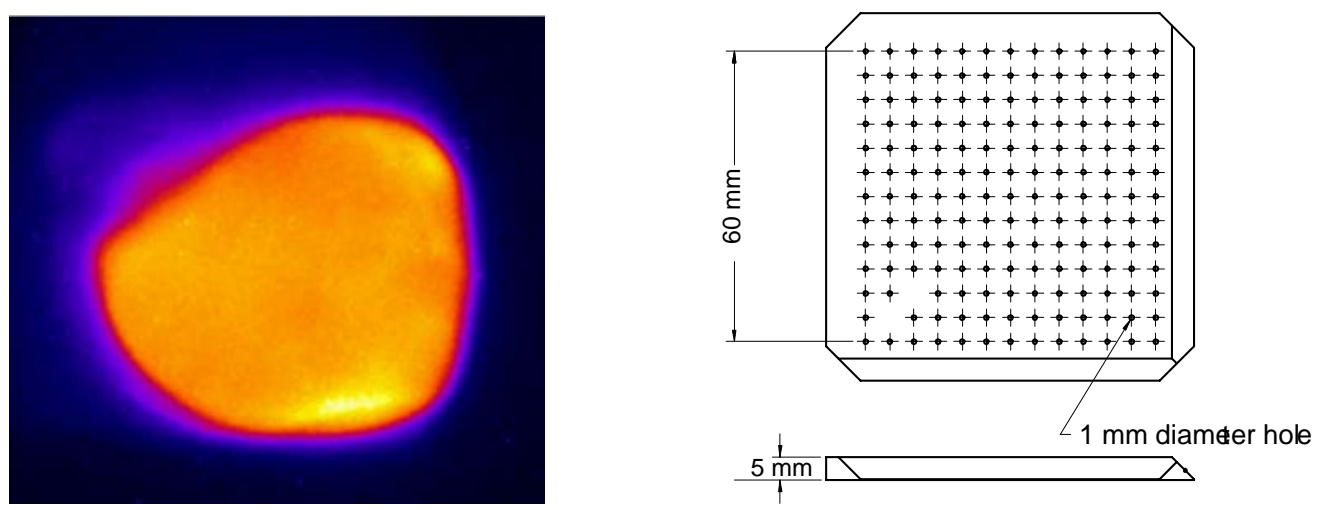

Figures 3 and 4. Image of the beam taken near the location of the mask, and a drawing of the $13 x 13$ mask. The image in Figure 3 was taken using a quartz plate (Cherenkov radation).

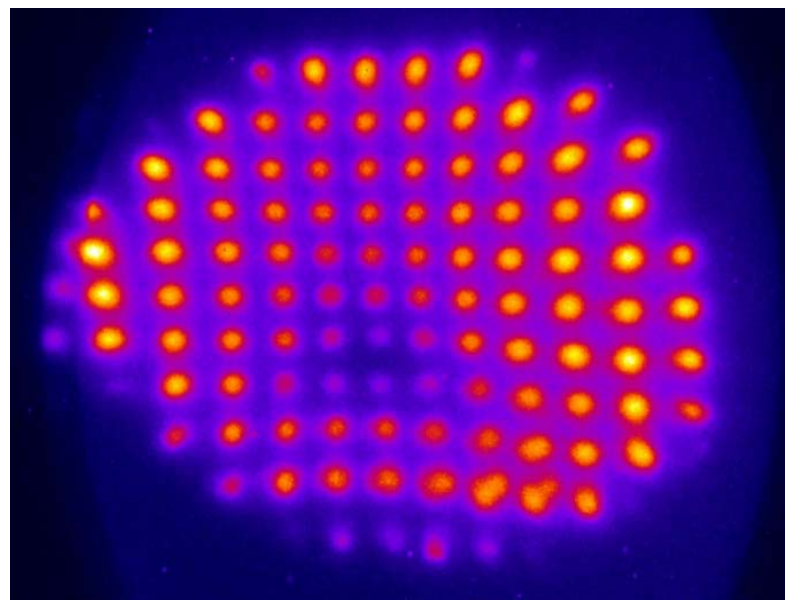

Figure 5. False color image of the beamlets on the Bicron Scintillator. (Shot 2828).

Figure 5 shows a pepper-pot image. There are 12 rows shown in the horizontal plane. From this image it is not certain that the edge of the beam was captured. However, other shots gave confidence that another row would not have shown beamlets. The Bicron Scintillator showed signs of transient darkening (to be discussed later) that produced the dimmer beamlets near the center region. 
To analyze the images we used a software program called Suicidetails ${ }^{2}$ (written by Frank Chambers and Brett Raymond). Earlier work by $\mathrm{Art} \mathrm{Paul}^{3}$ on the analysis of pepper-pot measurements on the FXR and ETA machines was useful in writing the code.

The first step in the analysis consist of mapping the beamlet images to holes in the mask. Figure 6 shows the assumed connections to the mask. The pattern displays both beam expansion/contraction and distortion.

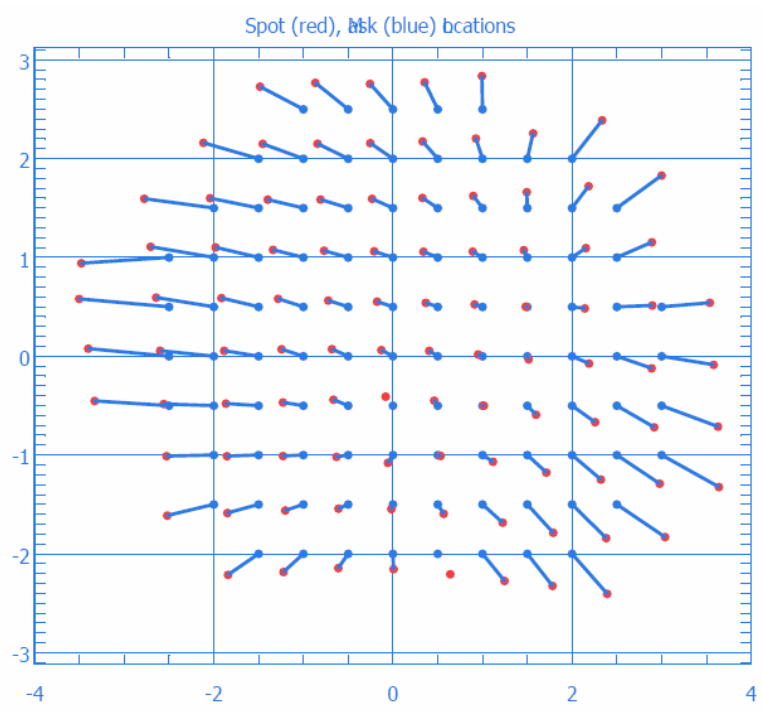

Figure 6. Mapping the beamlets in the image to holes in the mask. The red dots show the center of the beamlets. The blue dots are from the mask. The lines connecting the red and blue dots suggests the transverse velocity of the beamlets.

Let $x^{i}$ be the mask $x$ location of the $i^{\text {th }}$ beamlet and $v_{x}{ }^{i}$ be the $x$ velocity of the $i^{\text {th }}$ beamlet. The values of $x^{i}$ and $v_{x}{ }^{i}$ can be used to generate a "distortion" emittance number which does not include the thermal spread of the beamlets.

The next step is to analyze the spread of the beamlets. Figure 7 shows the width of each beamlet (red lines in the beamlet images). The width used in the program is from a 2D Gaussian fit to the image.

$$
I(x, y)=I_{0} \cdot \exp \left(-\frac{x^{2}}{2 \sigma_{x}^{2}}-\frac{y^{2}}{2 \sigma_{y}^{2}}\right)+I_{b a c k}
$$

Note: The program has 4 variables to fit the data $\left(\mathrm{I}_{0}, \mathrm{I}_{\text {back, }}, \sigma_{\mathrm{x}}\right.$, and $\left.\sigma_{\mathrm{y}}\right)$. The program is using an $\mathrm{I}_{\text {back }}$ obtained from the pixels in a box around the center of the beamlet. If the beamlets start to overlap it will cause problems with the analysis. The size of the box can be adjusted.

\footnotetext{
2 “Suicidetails System for Data Analysis, Management” by Frank Chambers \& Brett Raymond, presented at the Fifth Tri-Laboratory Engineering Conference, Oct. 21-23, 2003.

${ }^{3}$ A.C. Paul, et al., "ETA-II Beam Brighness Measurement”, PAC 1991
} 


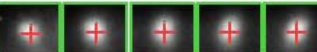

\begin{tabular}{l|l|l|l|l|l|l|l|}
$*$ & $*$ & $*$ & $*$ & $*$ & $*$ & $*$ & $*$
\end{tabular}

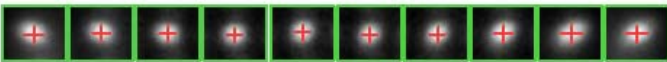

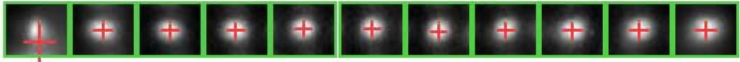

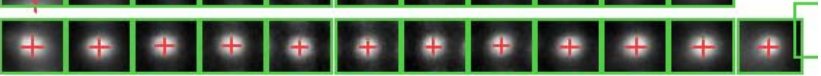

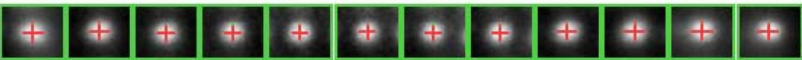

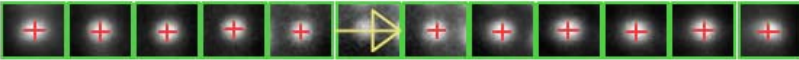

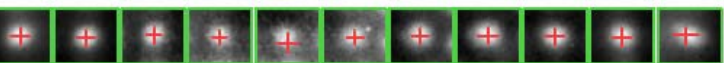

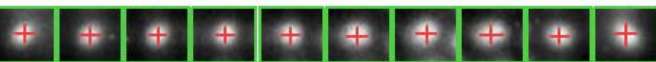

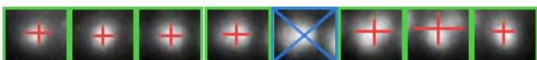

Figure 7. Width of the beamlets are shown by the red lines. Notice the program suggests a large width for a few of the beamlets. If a beamlet is distorted or approaches one of it's neighbors, the program has problems. The contribution of a beamlet to the emittance calculation can be removed, but then the emittance will be changed.

The value $\sigma_{\mathrm{x}}$ and $\sigma_{\mathrm{y}}$ returned are the width at which the fitted Gaussian has the value of 0.605 of the peak (See Figure 8)

$$
I\left(\sigma_{x}, 0\right)=I_{0} \cdot e^{-\frac{1}{2}}+I_{b a c k}=(0.605) I_{0}+I_{b a c k}
$$

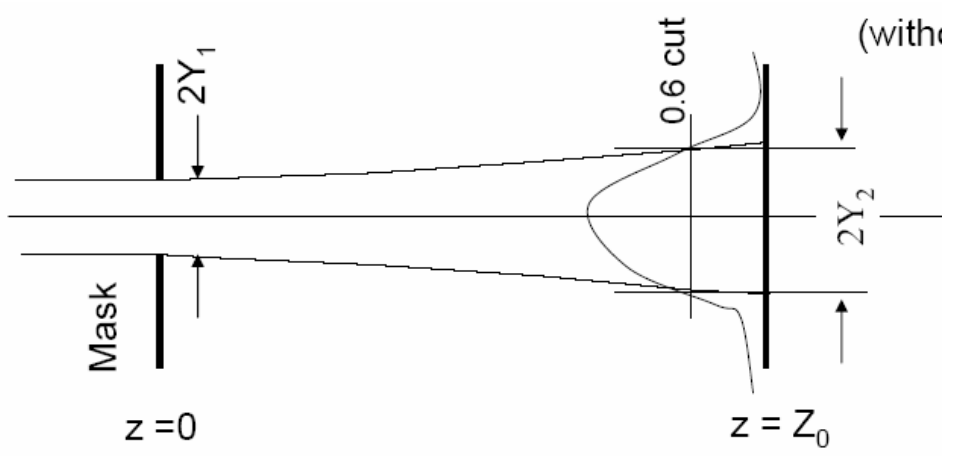

Figure 8. Expansion of the individual beamlets.

The next step is to calculate the beamlet's thermal spread. Consider the transverse expansion of a beamlet (relative to it's center). We assume the beamlet's $\mathrm{y}_{\mathrm{rms}}$ would be given by:

$$
y_{\text {rms }}^{2}(z)=Y_{1}^{2}+\left[\frac{\varepsilon_{\text {thermal }}}{Y_{1}}\right]^{2} z^{2}
$$


Where $\mathrm{Y}_{1}$ is the value at $\mathrm{z}=0$ and $\varepsilon_{\text {thermal }}$ is the beamlet's emittance as shown in Figure 8 . We only measure the radial size at $\mathrm{z}=\mathrm{Z}_{0}$. Just as the beamlet leaves the mask it will have a uniformly filled phase space with an edge diameter given by the hole diameter.

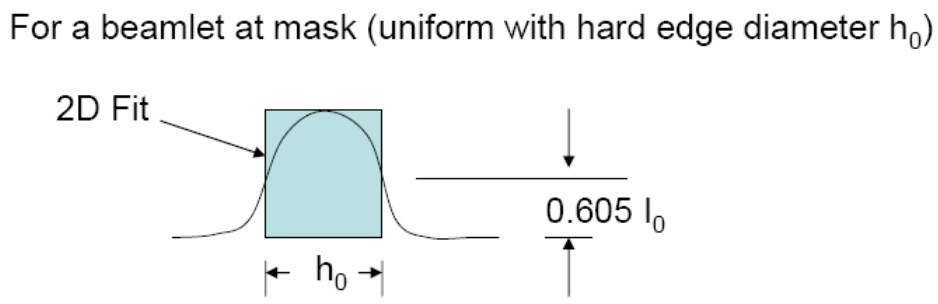

The method described above returns a $2 \sigma_{\mathrm{y}}=$ hole diameter. Therefore for $\mathrm{Y}_{1}$ we can use the radius of the hole. The value of $\theta_{\text {th }}$ can then be determined for each of the beamlets from the equation:

$$
Y_{2}^{2}(z)=Y_{1}^{2}+\theta_{t h}^{2} Z_{o}^{2}
$$

Knowing $\mathrm{Y}_{1}, \mathrm{Y}_{2}$ and $\mathrm{Z}_{\mathrm{o}}$ for each beamlets the $\theta_{\text {th }}$ can be plotted as seen in Figure 9 . There are a few point in Figure 9 that have $\theta_{\text {th }}>1.5 \mathrm{mrad}$. These correspond to beamlets in Figure 7 that have a large size. They typically result from beamlet distortion, or problems in resolving neighboring beamlets. For this case, it can be shown that removing them has only a minor effect on the final emittance number.

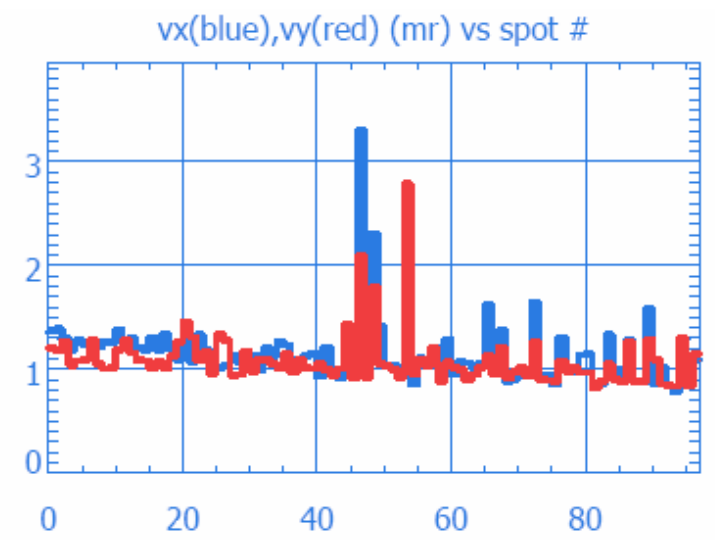

Figure 9. Thermal spread $\left(\theta_{\text {th }}(\mathrm{mr})\right)$ for the individual beamlets ordered by peak intensity.

The next step is to include the thermal spread in the emittance calculation of the total beam. The contribution of the beamlet to the $x-x^{\prime}$ phase space is represented by two points. The points from all the beamlets are then used to calculate the beam's emittance. The two points for the $\mathrm{i}^{\text {th }}$ beamlet are given by:

and

$$
\mathrm{x}=\mathrm{x}^{\mathrm{i}}, \mathrm{v}_{\mathrm{x}}{ }^{\mathrm{i}}=\mathrm{v}_{\mathrm{z}} *\left(\theta_{\mathrm{x}}{ }^{\mathrm{i}}+\theta_{\mathrm{th}}{ }^{\mathrm{i}}\right)
$$

$$
x=x^{i}, \quad v_{x}^{i}=v_{z} *\left(\theta_{x}{ }^{i}-\theta_{\text {th }}{ }^{i}\right)
$$


(where $\mathrm{x}^{\mathrm{i}}$ is the $\mathrm{x}$ location at the mask for the $\mathrm{i}^{\text {th }}$ beamlet and $\theta_{\mathrm{x}}{ }^{\mathrm{i}}$ is the transverse angle of the centroid of the $\mathrm{i}^{\text {th }}$ beamlet)

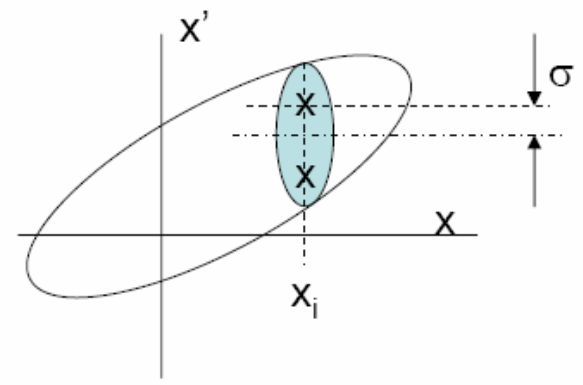

If the distribution in the transverse velocity is Gaussian, then this is exact.

$$
\text { For example if } \quad \rho(v)=\exp \left(\frac{-\left(v-v_{o}\right)^{2}}{2 \sigma^{2}}\right) \quad A_{0}=\int_{-\infty}^{\infty} \rho(v) d v
$$

then

$$
\left\langle v^{2}\right\rangle=\frac{1}{A_{o}} \int_{-\infty}^{\infty} v^{2} \rho(v) d v=v_{o}^{2}+\sigma^{2}=\frac{1}{2}\left[\left(v_{o}+\sigma\right)^{2}+\left(v_{o}-\sigma\right)^{2}\right]
$$

Figure 10 then shows the 194 points/plot, used in Shot 2828 (97 beamlets).

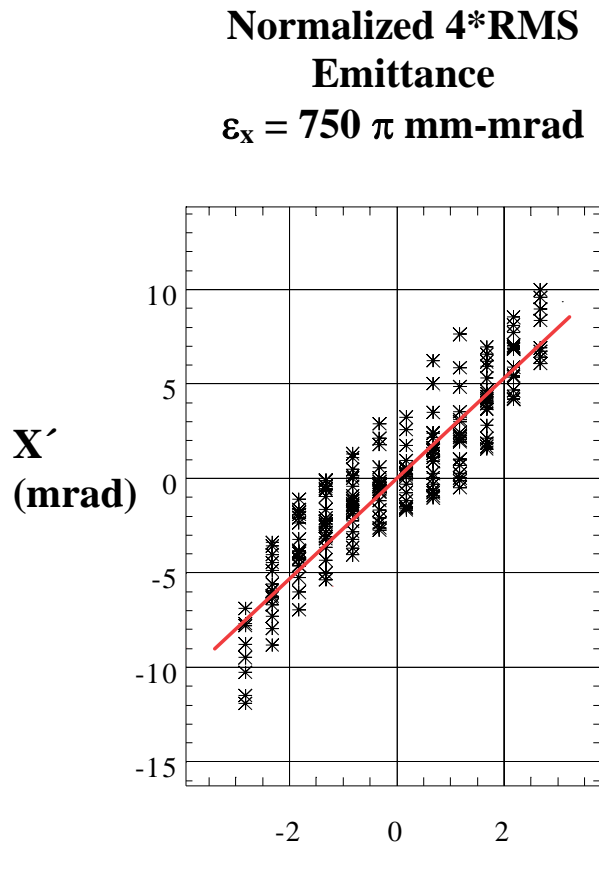

X position on mask
Normalized $4 *$ RMS Emittance $\varepsilon_{\mathrm{y}}=470 \pi \mathrm{mm}-\mathrm{mrad}$

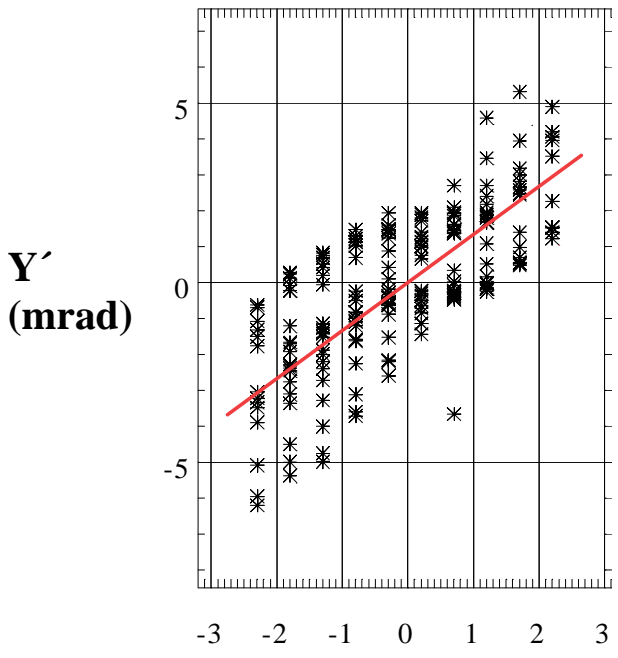

Y position on mask

Figure 10. $\mathrm{X}-\mathrm{X}^{\prime}$ and $\mathrm{Y}-\mathrm{Y}^{\prime}$ phase space for the beam. Red line shows the tilt. 
The rms values for the beam parameters can then be determined.

\begin{tabular}{|c|c|c|c|c|c|c|}
\hline xrms & $\mathrm{cm}$ & 1.66962 & & yrms & $\mathrm{cm}$ & 1.27943 \\
\hline vxbar & $\mathrm{mr}$ & $1.9645 \mathrm{e}-009$ & & vybar & $\mathrm{mr}$ & $9.7134 \mathrm{e}-009$ \\
\hline vxrms & $\mathrm{mr}$ & 4.68205 & & vyrms & $\mathrm{mr}$ & 2.19351 \\
\hline xvxbar & $\mathrm{cm}-\mathrm{mr}$ & 7.22222 & and & yvybar & $\mathrm{cm}-\mathrm{mr}$ & 2.07771 \\
\hline
\end{tabular}

And the emittance is calculated from these values:

$$
\varepsilon_{\mathrm{X}}^{2}=\langle\mathrm{X}\rangle^{2}<\mathrm{X}^{\prime}>^{2}-\left\langle\mathrm{xx}^{\prime}\right\rangle^{2}=\mathrm{xrms}^{2} \cdot \mathrm{vxrms}^{2}-\mathrm{xvxbar}^{2}
$$

This gives an unnormalized rms value. The emittance number typically quoted is the normalized $4 *$ rms emittance number $\left(\gamma \beta * 4 \varepsilon_{\text {rms,un }}=(25.2) * \varepsilon_{\text {rms,un }}\right.$ for these experiments $)$.

If only use one point per beamlet (at it center) then for this Shot \#2828 we get:

$$
\varepsilon_{\mathrm{x}, \text { distortion }} \sim 551 \pi \mathrm{mm} \text {-mrad, and } \varepsilon_{\mathrm{y} \text {, distortion }} \sim 303 \pi \mathrm{mm} \text {-mrad }
$$

We referred to this as the distortion emittance. The "thermal" emittance (from the spreading of the beamlets) can be obtained from:

$$
\varepsilon_{\text {total }}^{2}=\varepsilon_{\text {distortion }}^{2}+\varepsilon_{\text {thermal }}^{2}
$$

The thermal term for Shot \#2828:

$$
\varepsilon_{\mathrm{x}, \text { thermal }} \sim 504 \pi \mathrm{mm}-\mathrm{mrad} \text {, and } \quad \varepsilon_{\mathrm{y}, \text { thermal }} \sim 353 \pi \mathrm{mm}-\mathrm{mrad}
$$

The thermal term remained almost constant for all shots.

Since the beamlets have different currents, they should be weighted. When calculating the rms values, the points are weighted by the $\left(\sigma_{\mathrm{X}} * \sigma_{\mathrm{X}} * \mathrm{I}_{0}\right)$ terms in the Gaussian fit. This is related to the "volume" under the fit for a beamlet. This was done in almost all the analysis described in the report.

\section{Time Scans}

Most of the shots were taken early within the pulse. However there were 4 time scans performed during the experimental period. Figure 11 shows one of these scans (Scan \#3), and Figure 12 the emittance for Scan \#3 and Scan \#4. See Figure 13 for beam size near mask and Figure 14 for the injector voltage waveform. 


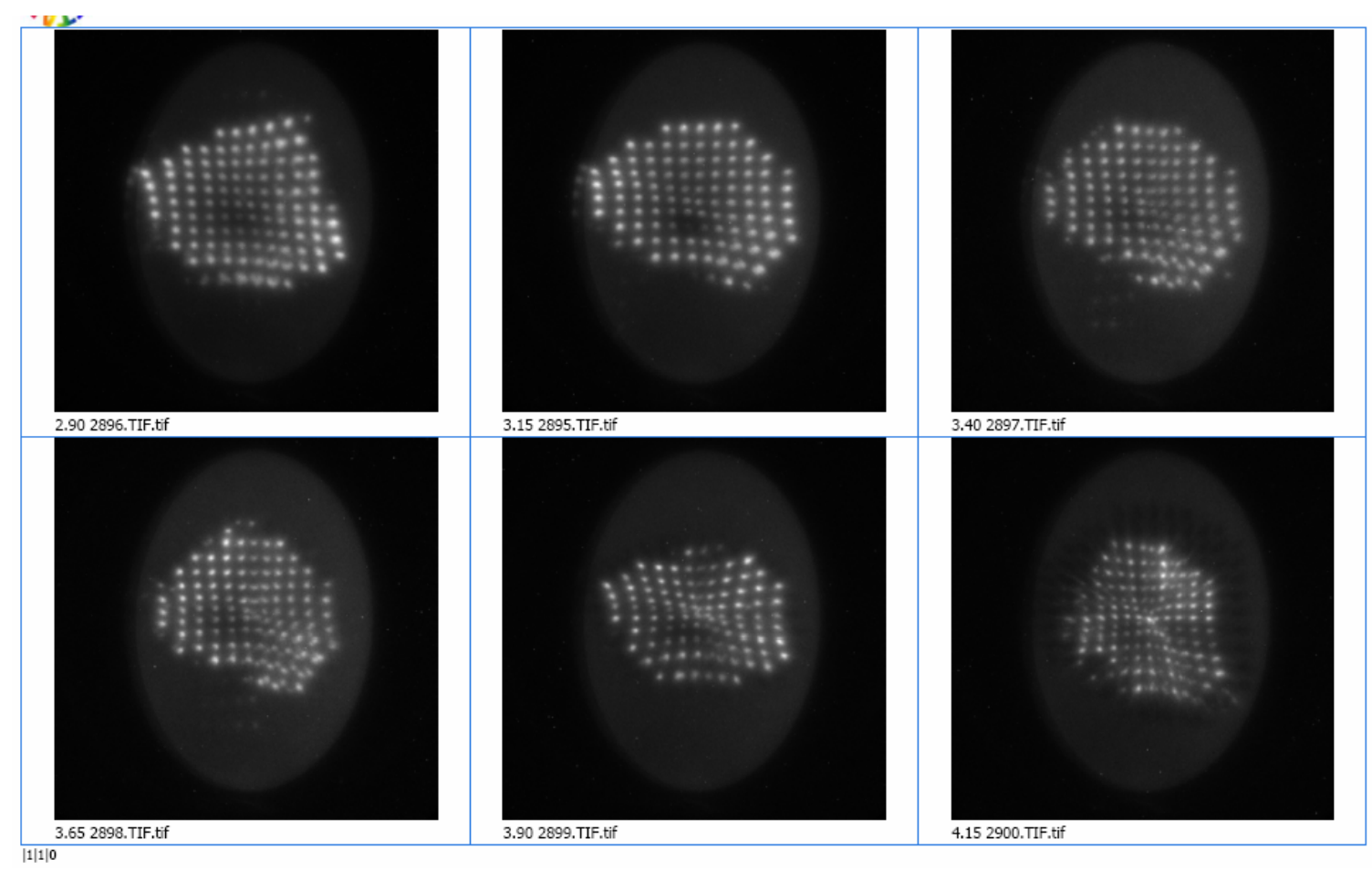

Figure 11. Image for Time Scan \#3 ; Shots 2895-2900 taken at times = 2.9, 3.15, 3.40, 3.65, 3.90, and $4.15 \mu$ s. At time $2.9 \mu$ s the voltage had reached its "flat top" level.

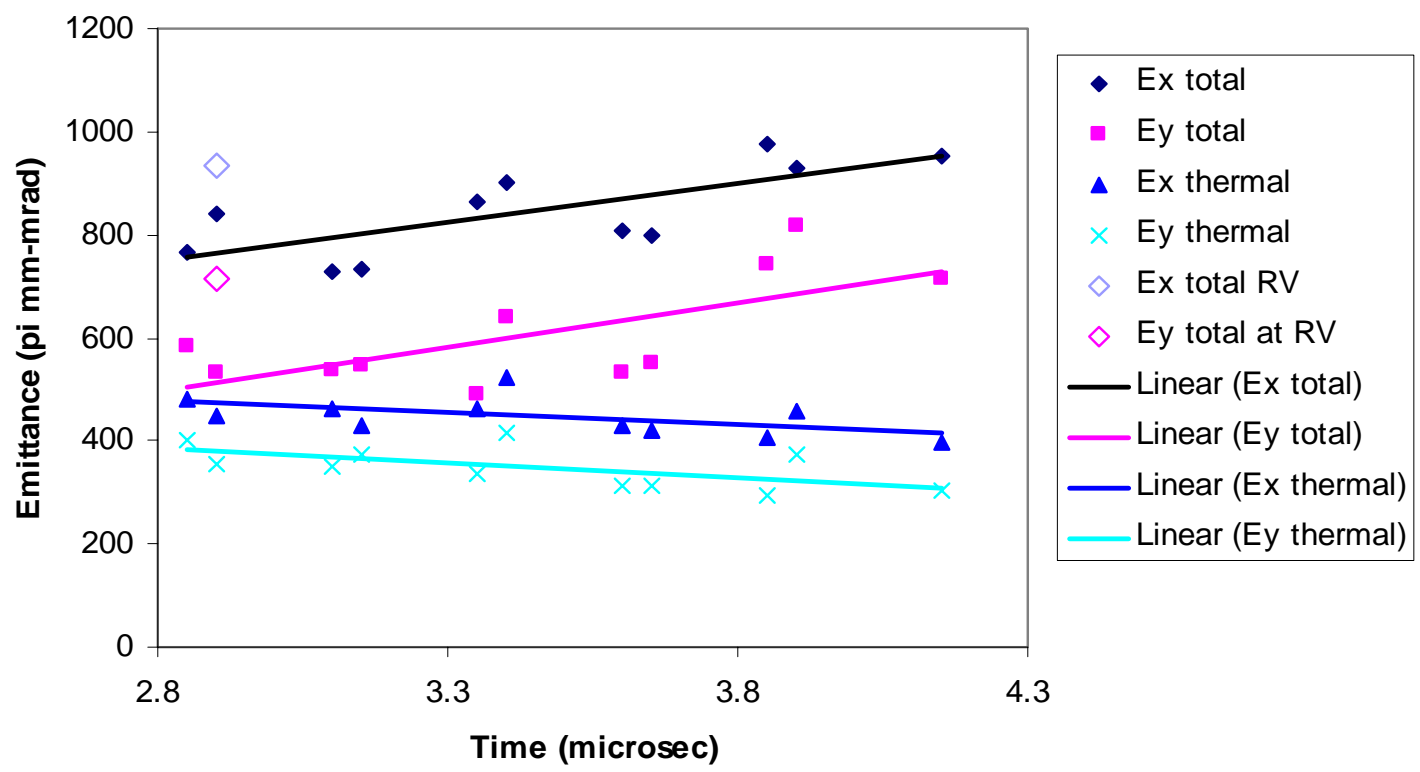

Figure 12. Results for Time Scan \#3 and Time Scan \#4. The single point entries labeled "Ex total RV" and "Ey total at RV" (last two on legend) are for a shot taken at $2.9 \mu \mathrm{s}$, but with a reduced voltage request for the injector (40 kV lower than normal). Scan \#3 times are shifted early by $0.05 \mu$ s to prevent overlap with Scan \#4. Expect for the "Ey total” point at $3.40 \mu \mathrm{s}$, the two scans produced almost the same curves. The Scan \#3 did not include a shot at $4.15 \mu \mathrm{s}$. 
We tried to determine if the change in the image size on the scintillator was a result of a change in the beam voltage, or from other effects. At the location of the mask the beam's radius was nearly constant (See Figure 13).

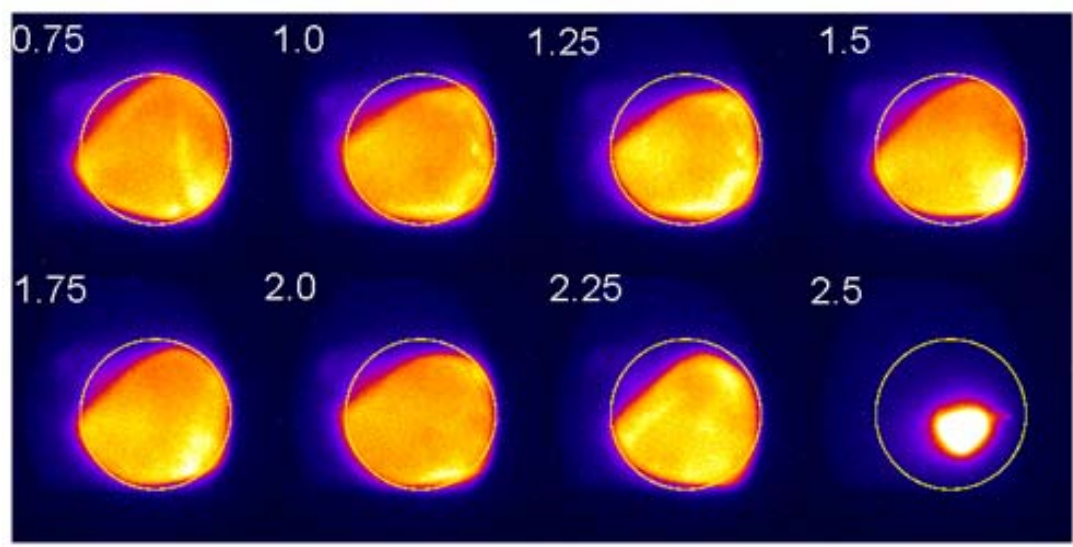

Figure 13. Beam size near the mask. Time shown should be shitted by $2.15 \mu$ s for comparison to other figures in this report.

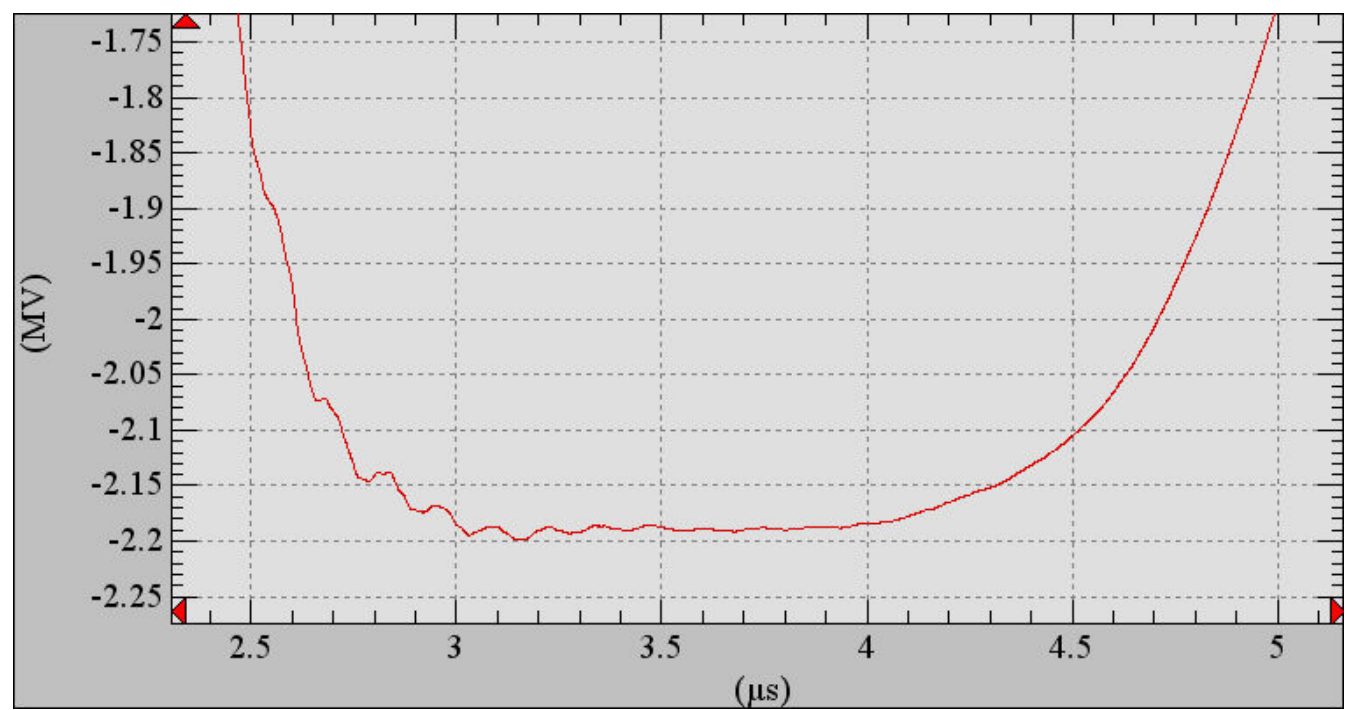

Figure 14. Voltage waveform for the injector. Shots shown in Figure 11 were from 2.9 to $4.15 \mu \mathrm{s}$.

The variations in the tilt at the mask (See Figure 15) produced the change in image size at the scintillator. 


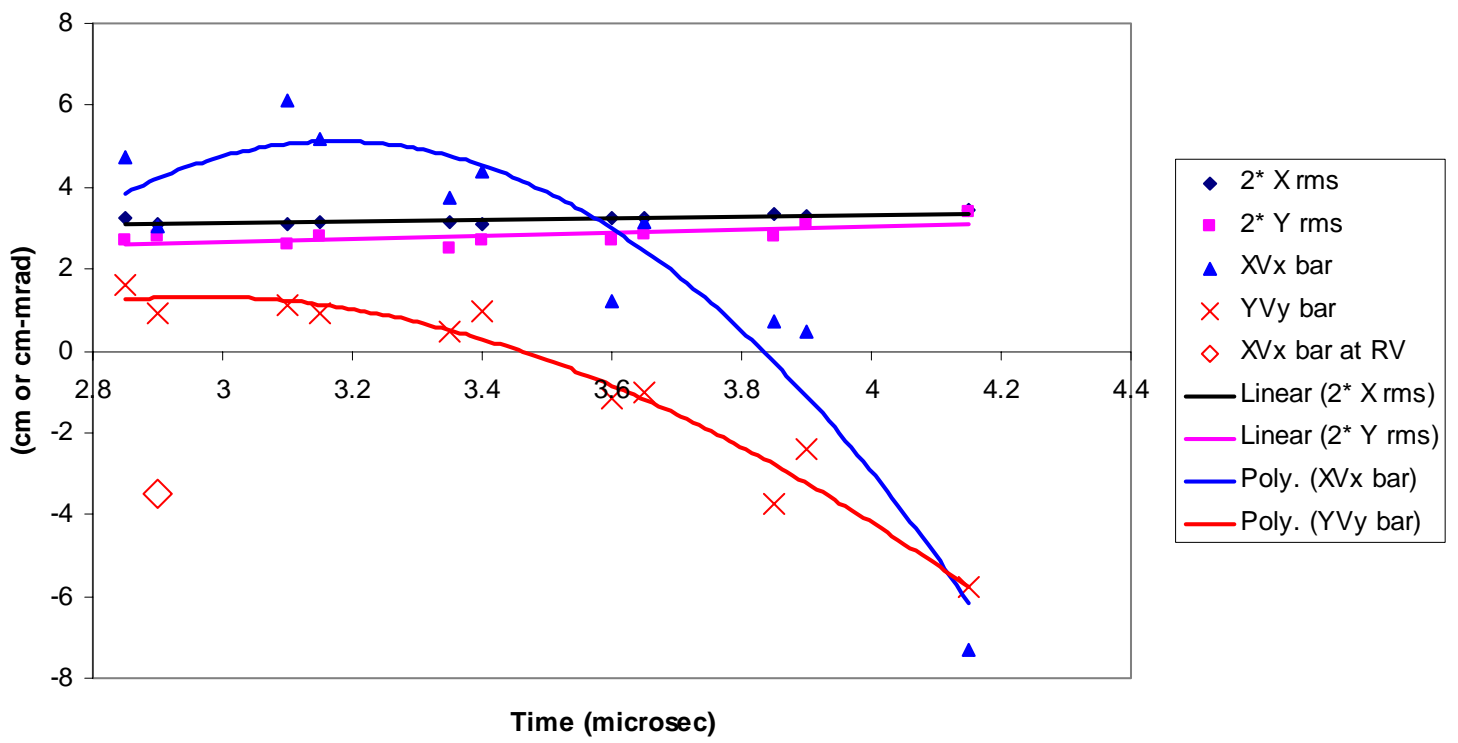

Figure 15. Radius and tilt of beam.

Most of the change in image size resulted from the reduced voltage. The tune had a narrow waist in front of the mask (see latter section). The position of the waist was very energy sensitive. If the beam's voltage had been flat, we would expect little change in the beam’s emittance.

\section{Late-time plasma effects}

The images taken late in the pulse were difficult to analyze because of beamlet distortions near the center of the image.

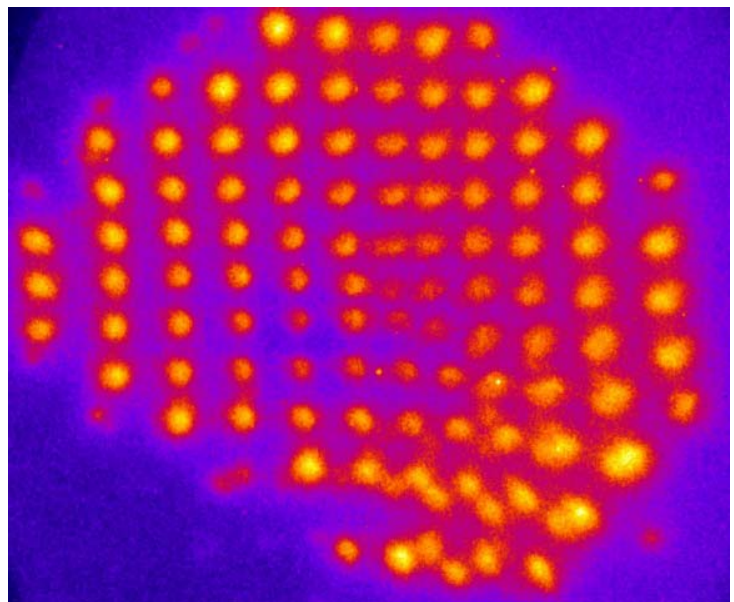

Figure 16. Typical of early time (Shot 2897). 

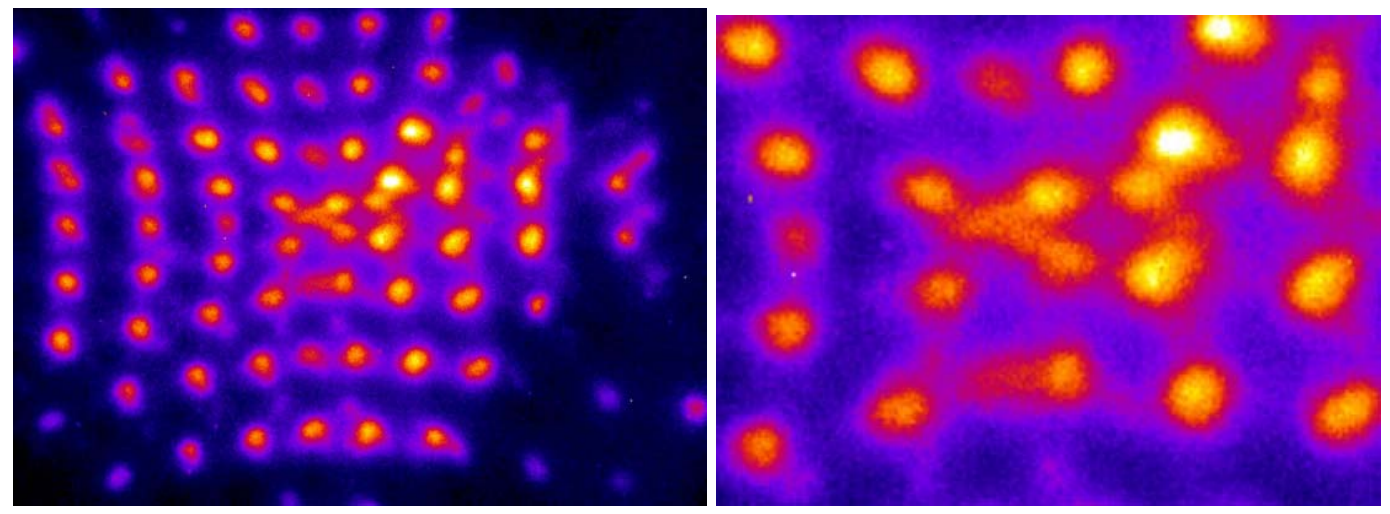

Figure 17 and 18. At late time (Shot 2876). Second is a blow up of the center region.

Ions released by the mask will fall into the potential well created by the beam ${ }^{4}$. They would be located near the beam's center. This would cause a larger effect on the trajectories of the center beamlets at later times (needs to shown by simulation).

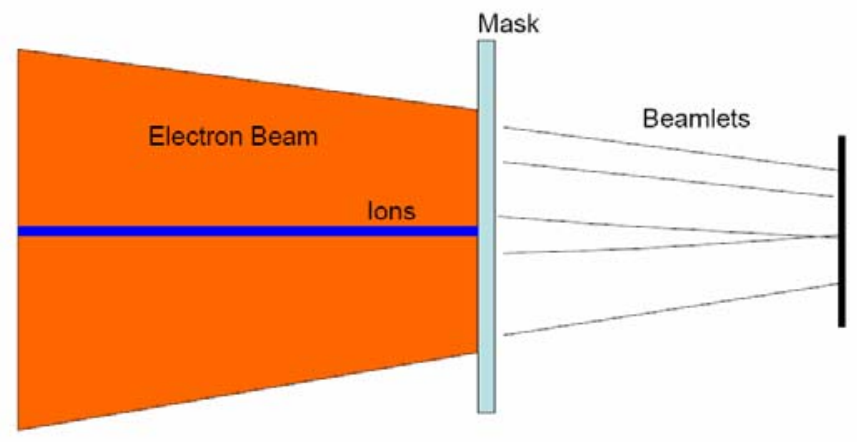

Figure 19. Possible explanation of distortion of center beamlets.

The late-time shots show evidence of transient darkening that complicate the analysis.

\section{Bucking Coil setting}

We took one shot with the bucking coil set at 7.0 A (Shot \#2851) instead of the normal 8.1 A setting. This seemed to rotated the observed pattern by about $90^{\circ}$ (See Figure 20). Analysis showed $\varepsilon_{\mathrm{x}}<\varepsilon_{\mathrm{y}}$ for this shot. This may suggest that the larger value for $\varepsilon_{\mathrm{x}}$ in the typical case results from forces near the cathode surface, or from non-uniform emission of the cathode. The values for $\varepsilon_{\mathrm{x}}$ and $\varepsilon_{\mathrm{y}}$ are large for this shot. The change in the magnetic field on the source effects the beam radius and the beam radial displacement throughout the machine.

\footnotetext{
${ }^{4}$ Suggested by Y-J Chen
} 


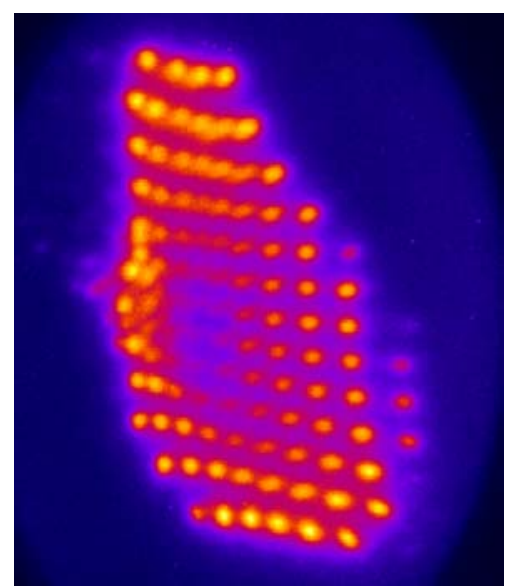

Figure 20. Pepper-pot image with low bucking coil setting.

\section{September 2005 experimental run}

Pepper pot measurements were also taken during September 2005. There were 6 injector cells and 50 accelerator cells in the systems at this time period. The beam energy at the pepper pot was 7.1 MeV. For a more complete description of other measurements taken during the September run see Appendix A.

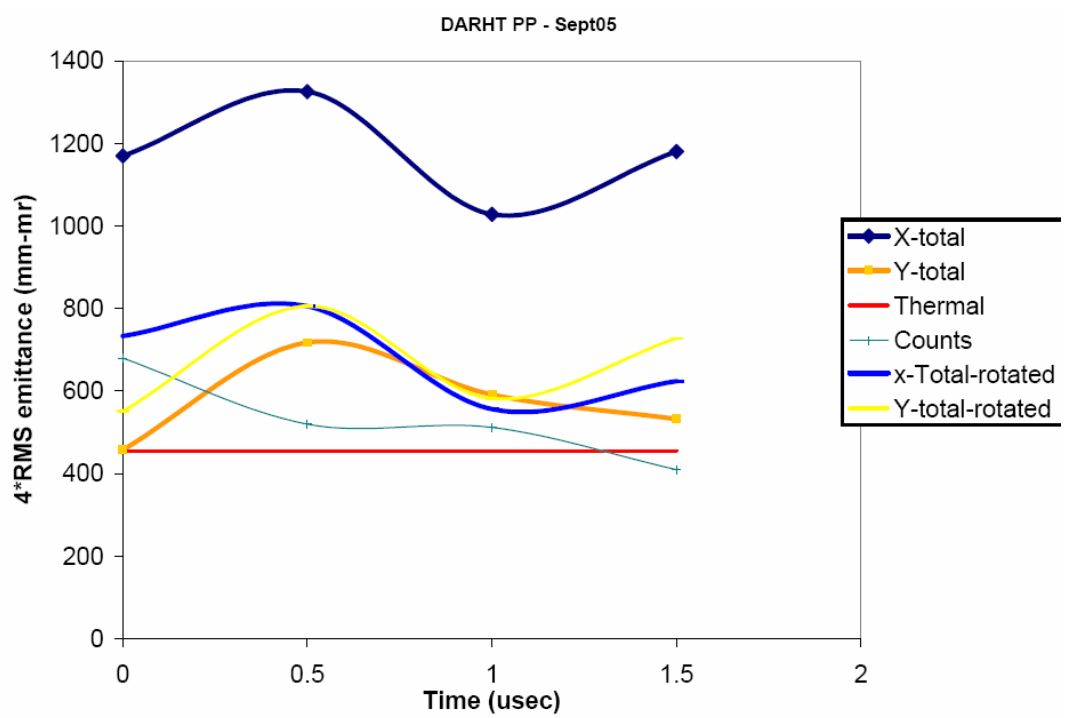

Figure 21. Emittance in the September experiments.

They were also taken using a different camera than the January 2006 set. The camera used did not have the resolution of the new camera, and there was more noise in the image. The mask hole size was only 2 pixels, and the expansion was 1 or 2 pixels (see Figure 22). This does not allow for a good measurement of the expansion. However, the average thermal emittance measured was $\sim 450 \pi \mathrm{mm}$-mrad, which was comparable to the January thermal emittance number. 


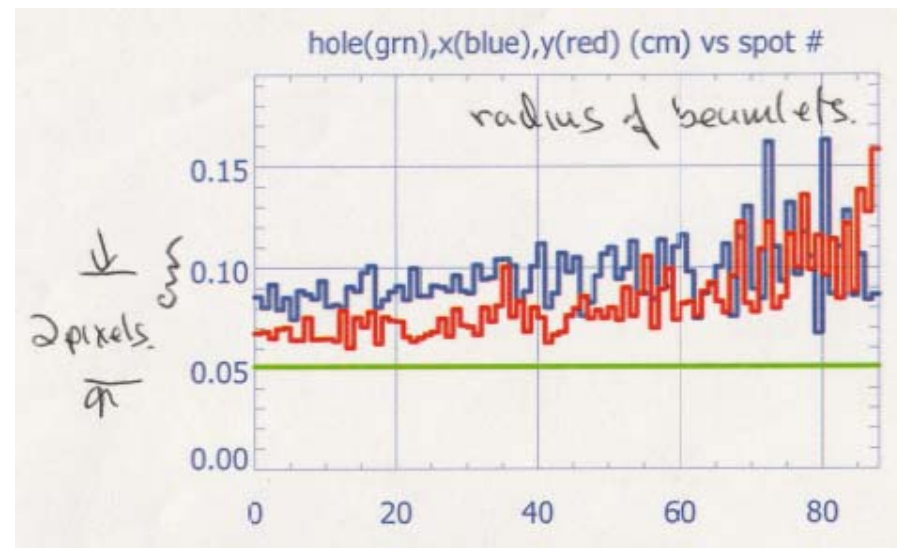

Figure 22. Expansion was difficult to determine with the pixel resolution used in the September experiments.

The transverse velocity of the beamlets indicated a pattern (See Figure 23) that could be created by a rotation and a quadruple moment.

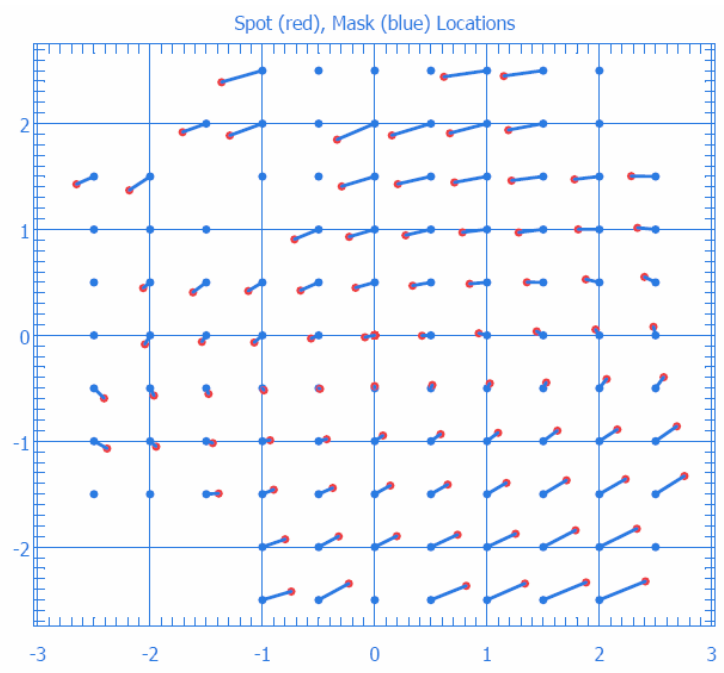

Figure 23. Pattern of transverse velocity in the September experiment.

The emittance in the horizontal plane (x) was larger than the vertical plane (y).
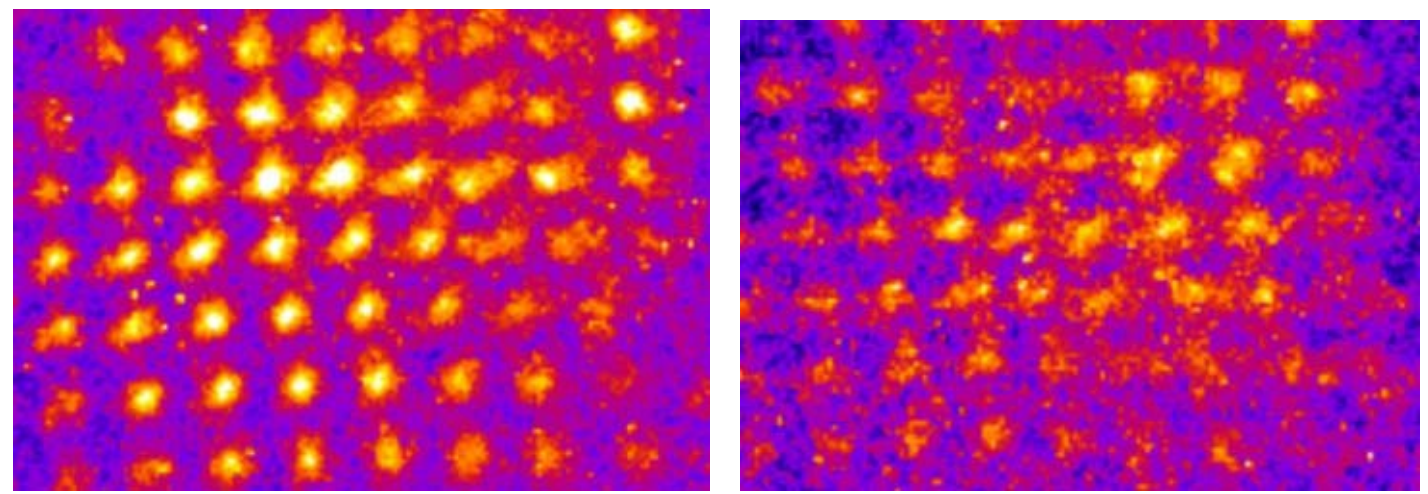

Figure 24. Early and late time images for the September 2005 run. 
We were less sensitive to kicks near the mask because of the higher beam energy. Also as can be seen in Figure 24 the image quality was much poorer. It would have been harder to see any effects from the back-streaming ions.

\section{Beam Tune}

Figure 25 shows our best guess for the beam envelope for the tune used for the pepperpot measurements. It was generated from the conditions measured at the mask $(\mathrm{z}=800$ $\mathrm{cm}$ ) and the currents to the solenoids. The beam voltage was assumed to be $2.7 \mathrm{MeV}$.

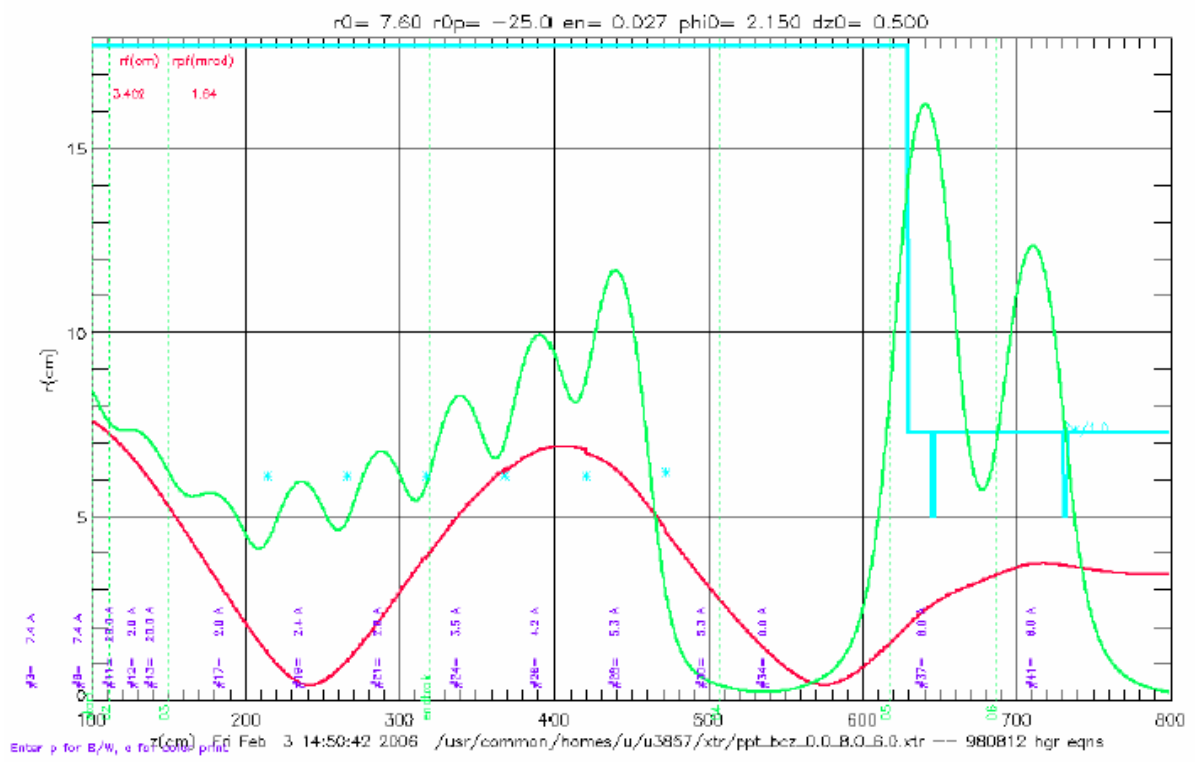

Figure 25. Beam envelope shown in red. (generated by Enrique Henestroza). The horizontal axis is $\mathrm{z}(\mathrm{cm})$, and the vertical axis is $\mathrm{r}(\mathrm{cm})$. The pepper pot mask is located at $800 \mathrm{~cm}$. The magnet field is shown in green.

We should try a tune where the beam does not go through tight waist. The tight waist can result in an increase in the emittance for a non-uniform density beam.

\section{Steering the beam}

Use of steering to bring the beam onto the accelerator axis seemed to result in beamlet distortion. Beside the dipole field they will also have generated fields with higher-order modes. At a radius of $6 \mathrm{~cm}$ MAFCO predicts $^{5}$ that

$$
(\text { Dipole Term }) /(\text { Sextupole Term })=80
$$

\footnotetext{
${ }^{5}$ Communications with Bill Fawley
} 
for the accelerator-cell steering elements (wall radius $12.7 \mathrm{~cm}$ ). The sextupole fields have $\mathrm{r}^{2}$ dependence. If the beam edge approaches the wall in a region where one is using these steering elements, there can be higher mode introduced into the problem.

\section{Space Charge Forces}

In the analysis we ignored the space-charge forces in the expansion of the beamlets. From the envelope equation we want the space-charge term to be small compared to the emittance term.

$$
\begin{aligned}
& \text { Envelope equation } \quad \boldsymbol{R}^{\prime \prime}=\frac{2 I}{(\gamma \beta)^{3} I_{o}} \frac{1}{R}+\frac{\varepsilon^{2}}{\boldsymbol{R}^{3}} \\
& \text { want } \quad \frac{2 I}{(\gamma \beta)^{3} I_{0}} \stackrel{?}{<}<\left(\frac{\varepsilon}{R}\right)^{2}
\end{aligned}
$$

From the square of the ratio of the beam radius to mask hole size, we expect the current in a beamlet to be about $0.3 \mathrm{amp}$. If the thermal spread is 1 mrad then the space-charge term is about $1 / 6$ the emittance term for a location near the mask.

\section{Transient darkening}

A region in Figure 5 near the center is dimmer than expected. This dim region did not move with the mask or the screen. It was not seen for a diffuse beam. During the lowvoltage head of the pulse the beam can dwell on a small region of the mask. Scintillators using "color center" are prone to transient darkening effects when used with intense sources. The light output will diminish in time for a constant beam hitting the material. The reduced light output of the dim beamlets will decrease their weight to the final emittance. Turning the weighting feature off for Shot \#2828 reduces $\varepsilon_{\mathrm{x}}$ by $9 \%$ and $\varepsilon_{\mathrm{y}}$ by less than $1 \%$.

For the images in a time scan, if one sums the pixels within a region that includes all the beamlets, it shows that the latter shots have less total intensity. This is consistent with transient darkening phenomena.

\section{$\underline{\text { Return current bars and Foil Focusing }}$}

During the experiment we thought that the return current bars on the cross in front of the mask where the cause of the "octople" pin-cushion seen in the images (see Figure 26 for an example). However, simulations (Henestroza) indicate the effect should be small (small lever arm). Also, as was pointed out by Bieniosek, they were in the wrong direction. Moving the centroid positions with an "octopole field" after the mask did not significantly reduce the value of the emittance value. We also see indications of quadrupole (ellipitical beam), sextupole (see Figure 13, image of the beam at the mask) modes. The beam is getting kicked hard, and generating distortions for all the "modes". 


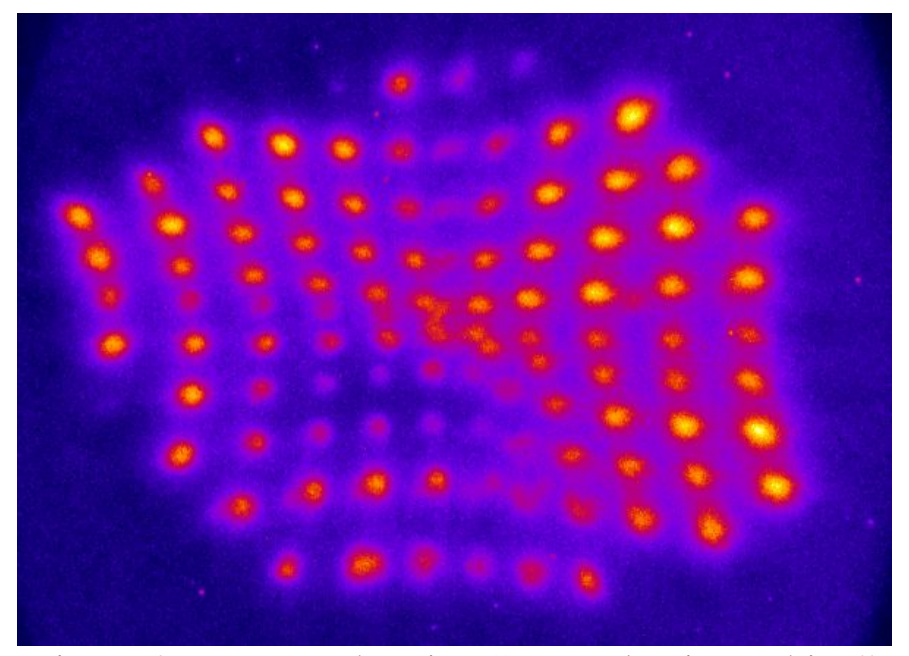

Figure 26. Image showing “octopole pin-cushion”

We do not believe that foil focusing had much effect on the emittance number. In simulations the effect of changing between Neumann and Dirichelet boundaries for the mask on the emittance was shown to be neglibible. The beam size did not change, however the convergence was reduced by $\sim 10 \mathrm{mrad}$.

\section{Removing beamlets from an analysis}

When the fitting routine for a beamlet failed, its contribution to the emittance was dropped. For example the center beamlets in Figure 25 cannot be resolved. The beamlet for some mask holes cannot be determined. The width of the beamlets is even harder to determine, especially when they split or approach neighboring beamlets. In most cases the final emittance is not sensitive to the "dropping" of many of the beamlets. For example, about 2/3 of the beamlets can be randomly removed in Shot \#2828 before the calculated emittances changes more that $20 \%$. It does require some number of beamlets to determine the beam's radius at the mask. However, beamlets created at edge of the beams which have "non-linear" velocity terms to contribute significantly to the emittance. How they will impact the final x-ray spot size is difficult to analyze.

\section{Effects not included}

( $\downarrow$ decrease the value of emittance, $\boldsymbol{\uparrow}$ increase the value of emittance)

1. Not capturing the edge of beam at the mask. ( $\uparrow$ )

In some of the images the edge of the beam is not captured. Having more mask rows would help reduce effect, but cause problems with overlapping images. Neglecting beamlets on the beam's edge will underestimate the beam's emittance.

2. Hooking of the phase space. ( $\boldsymbol{\uparrow}$ )

Many of the beamlets created at the beam's edge seem to be distorted. The program has problems fitting these images, and they are often emitted from the analysis.

3. Transient darkening of the Bicron material. 
See section on transient darkening above.

4. Gas focusing (near mask)

Gas will be desorbed near the mask. Generating an ion-channel for an individual beamlets will reduce its expansion rate. This could be caused by gas in the mask hole. Gas focusing in front of the mask will change the beam's emittance.

6. Motion of beam from $8 \mathrm{MHz}$ injector mode.

Using a 5-ns camera window reduced the effect. For 10 and 20 ns windows evidence of blurring can be clearly seen in the pepper-pot images. A 5-ns window includes $\sim 15^{\circ}$ of the $8 \mathrm{MHz}$ period.

7. Not including contribution of halo particles ( $\boldsymbol{\uparrow})$

Having a few particles with high $x$ and $x^{\prime}$ can raise the emittance. However they will not show as beamlets, and are ignored in the calculation. Their effect for the final application needs to be considered using other methods.

8. Space-charge expansion of the beamlets $(\boldsymbol{\downarrow})$

See section on space-charge effects above.

9. Vignetting of beamlets by the mask.

The mask holes may not have the acceptance to pass all the particles in the beamlets. The effect was reduced by using a thin mask.

10. Reemerging electrons by scattering at edge of holes.

Particle entering the carbon mask material can be scattered back into the beam.

They will increase the thermal spread of the beamlet.

11. Background scatter of electrons by mask.

Since the mask is range thin most of the beam will pass through the mask.

However, most have undergone scattering events, and the beam will rapidly

expand after leaving the mask. A number will hit the screen. However, we expect the flux on the screen of these electrons will nearly uniform. They will add to the background, but should not impact the emittance calculation.

12. Analysis simplification.

The analysis reduces complexity to determine the emittance.

\section{Code reliability}

We have tried to check the code used for calculating the emittance. However, there are a great number of variables. It was sometimes confusing what factors to include in the analysis (i.e. expansion rate). We have tried to compare it with past experiments on ETA analysis by A.C. Paul. We could reproduce his results, put needed to adjust the tool (i.e equally weight of beamlets, cut level used). Frank Chambers generated computer images and could recover the emittance used to generate the images. It would be useful for code simulators to generate pepper-pot images, to see if we recover their expected emittance. This would also help clarify that we are using the same definitions for the terms. 


\section{Acknowledgements}

Accelerator operations was directed by Trent McCuistian during the measurements. Work was encouraged by Ray Scarpetti and Simon Yu. Discussion with Carl Ekdahl during the experimental run were very useful in understanding the results.

The new camera used for these experiments provide higher quality images than was possible with previous cameras. Howard Bender helped to improve its performance. Angela Tipan and Carl Carson assisted in the camera setup and in acquiring images.

Brett Raymond helped support the analysis tool and helped to analysis the results. Discussion with Art Paul were useful in the development of the software. Martin Schauer assisted with the optics and provided the scintillator material. Bill Ghiorso did the mechanical design of the measurement hardware.

\section{Conclusion}

- Normalized $4 *$ rms emittance is $\varepsilon_{\mathrm{x}} \sim 800 \pi \mathrm{mm}$-mrad and $\varepsilon_{\mathrm{y}} \sim 600 \pi \mathrm{mm}$-mrad at early times. If voltage was $2.45 \mathrm{MeV}$ then $\varepsilon_{\mathrm{x}} \sim 740 \pi \mathrm{mm}$-mrad and $\varepsilon_{\mathrm{y}} \sim 550 \pi \mathrm{mm}-$ mrad.

- Beamlet expansion was almost constant for all cases, and the contribution to the emittance from the thermal expansion term was smaller or comparable to the optics (distortion) term.

- Although the emittance value changed as a function of time, we believe that the observed change can be explained by the variation in beam voltage and by ionfocusing (due to ions released at the mask).

- The data suggest that the beam is born with $\sim 30 \%$ more emittance in the $\mathrm{x}$-plane (Rotational experiments \& lack of cases with $\varepsilon_{\mathrm{y}}<\varepsilon_{\mathrm{x}}$ ). This may be non-uniform emission or an early kick in the injector.

- The emittance is higher than expected from computer simulations. Non-uniform emission and optics could produce the emittance growth when combined with :

- Tight pinch.

- Large beam radius change within a solenoid.

- Steering was detrimental to beam optics

- Large R (beam near wall) near steering element.

- Solenoid or B-dot misalignment.

- Field errors in solenoids.

- Field errors in steering elements. 
Preliminary progress report on the DARHT-II pepper pot experiments, August-

September 2005

\section{Frank Bieniosek, Enrique Henestroza, and the DARHT team}

September 28, 2005

The purpose of the pepper-pot experiment is to gather information on the 4dimensional transverse phase space of the DARHT-II electron beam. The area enclosed by the phase space of the beam is directly related to the achievable beam spot size on target. The two-dimensional projection of the phase space, characterized by the emittance, is a measure of the phase space area. The beam emittance is determined initially by the beam size and temperature at the injector. Emittance growth may occur throughout the accelerator. Measuring the emittance at the downstream location of the accelerator provides an upper bound on the emittance at the injector; further measurements are planned at a location close to the injector for detailed study of the beam as it exits the injector.

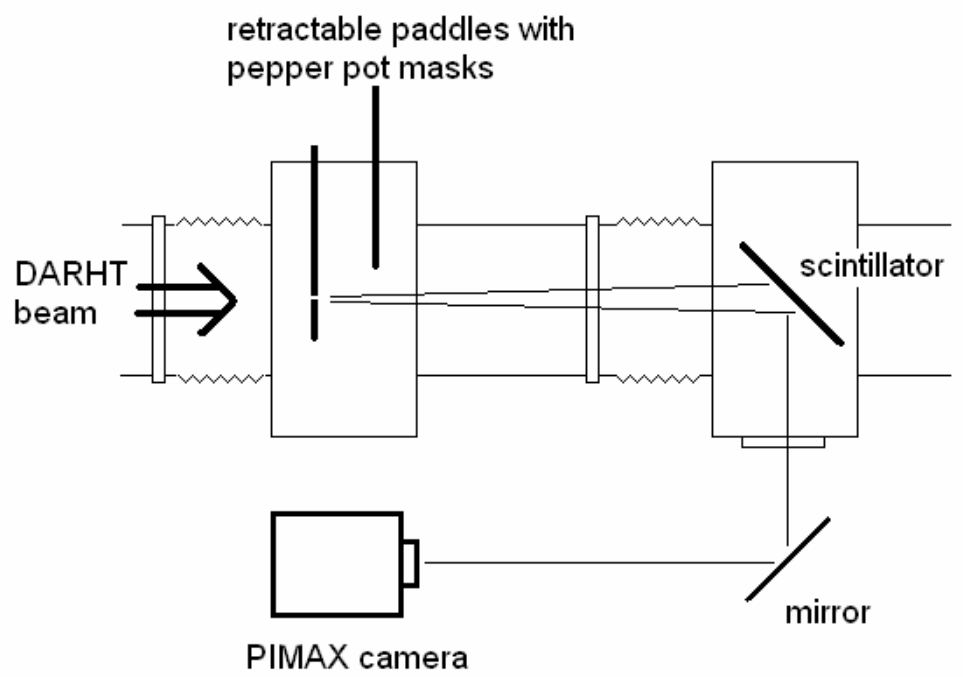

Figure 1. Layout of the pepperpot experiment.

The pepper-pot emittance diagnostic is based on similar experimental measurements performed on ATA [Ref. 1] and on ETA-II [Ref. 2]. The layout of the DARHT-II pepperpot diagnostic is indicated in Figure 1. A diagnostic tank was installed upstream of the imaging station located near the end of the accelerator. Two retractable paddles may be inserted into the beam path. Various patterns of 1-mm diameter holes were used in the graphite masks; two masks in the upstream paddle, and one graphite mask in the downstream paddle. The hole pattern was imaged at a 45-degree Bicron 422 scintillator at the imaging station, centered at $107 \mathrm{~cm}$ downstream of the upstream paddle. In addition, at the downstream location a 3.5-inch diameter quartz imaging plate was installed. In this case the beam cross section was imaged using a 45 -degree mirror at 
the imaging station. Images were taken using a Roper Scientific (Princeton Instruments) PI-MAX high speed image intensified camera, gated to a 10-ns time window which could be varied through the 2-ns DARHT-II beam pulse. The time window was gated to $10 \mathrm{~ns}$, which is the smallest time window available on this camera, because of corkscrewing in the beam at the $8-\mathrm{MHz}$ injector oscillation frequency and other frequencies. The corkscrewing is clearly evident by crescent-shaped beamlet images in a 50-ns window.

We used the graphite pepper pot mask to make a determination of the phase space distribution of the DARHT-II electron beam. The graphite mask was $0.95-\mathrm{cm}$ thick, which is about half of the range of the 7.1-MeV (kinetic energy) electron beam. The scattering of electrons passing through the mask was estimated from the equation [Ref. 3]:

$$
\theta_{0}=\frac{14.1 M e V / c}{p \beta} Z_{\text {inc }} \sqrt{\frac{L}{L_{R}}}\left[1+\frac{1}{9} \log _{10} \frac{L}{L_{R}}\right]
$$

where $L_{r}$ is the radiation length, about $18.8 \mathrm{~cm}$ in graphite. At a momentum of $p=7.6$ $\mathrm{MeV} / \mathrm{c}$, the RMS scattering angle is $\sim 350 \mathrm{mrad}$, thus over a drift distance on the order of 1 meter, the electrons scatter into a cone of 35-cm RMS radius. The scattered electrons provide a roughly uniform background on the $12-\mathrm{cm}$ diameter scintillator imaging the beamlets.
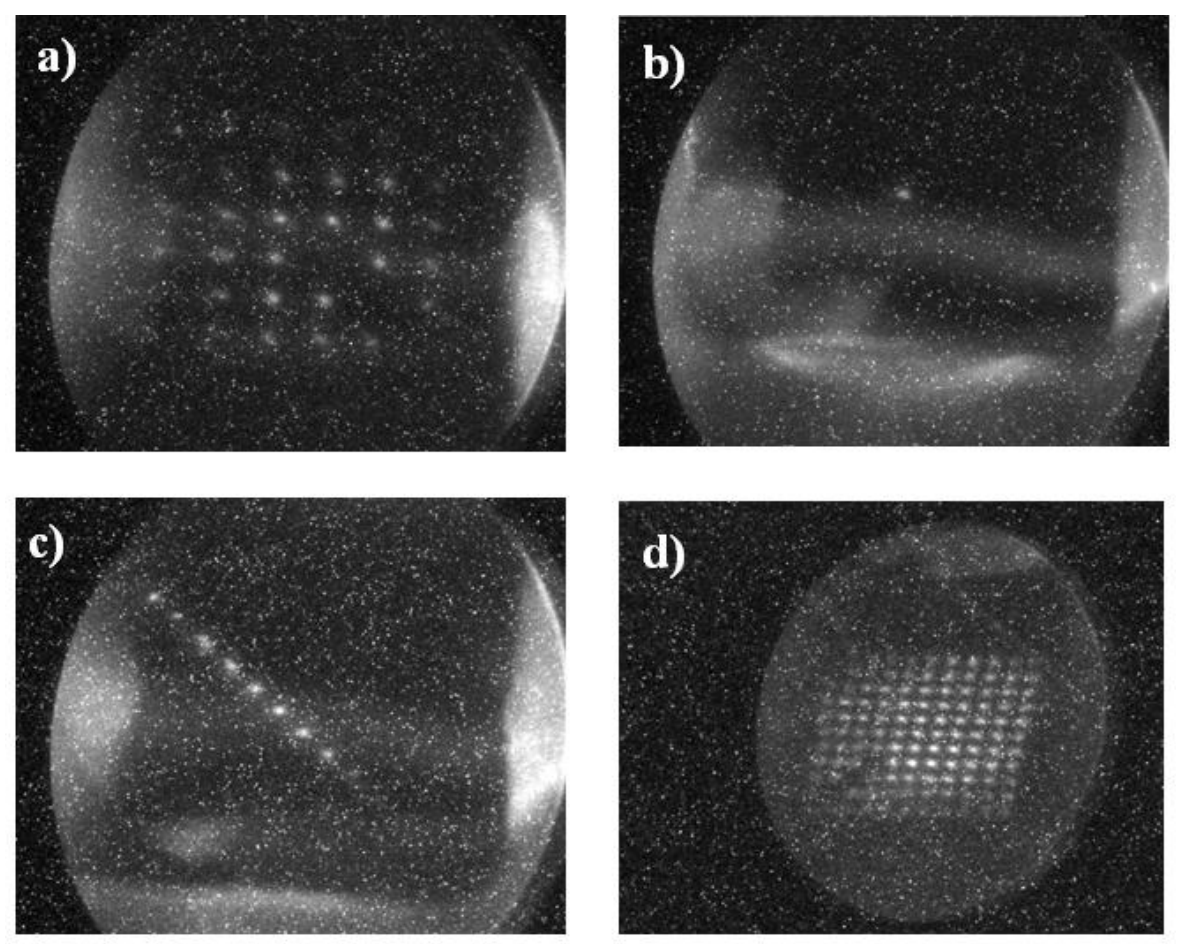

Figure 2 Beam imaged through various pepper pot mask configurations: a: $6 \times 6$ square hole array (10-mm spacing), b: single hole, c: diagonal hole pattern (7-mm spacing), d: $11 \times 11$ square array (5-mm spacing). 
Electrons pass through the pepperpot holes and continue to the scintillator deflected by their local angle of incidence; the image spots spread out by the transverse thermal spread of the electron distributions. The effect of space charge on the beamlets is negligible. Because of the long aspect ratio of the holes (1- mm diameter and 9.5-mm length) care must be taken to minimize the overall convergence/divergence angle of the beam at the mask location.

Thermal heating of the graphite mask by the incident electron beam is within acceptable limits [Ref 4]. Efforts were made to operate with a tune that provided a beam spot of about $3 \mathrm{~cm}$ radius to minimize surface heating and subsequent space charge neutralization by gas released from the surface of the mask.

Images of the beam through various pepper-pot masks are shown in Figure 2. Note that the square hole arrays have missing holes to provide a key for identifying the precise location of the beam in the pepper-pot array.

Note the presence of background due to several causes. There is x-ray background on the CCD camera chip, electrons scattered on passing through the mask cause the scintillator to light up roughly uniformly, and there is an enhanced background in Fig 2a through 2c because the beam is wider than the paddle holding the masks. In Fig $2 \mathrm{~d}$ the paddle has been widened to block the portion of the beam that passes through in Figs 2a through $2 \mathrm{c}$.

\section{Image processing}

Image processing was done for the most part using the Java-based scientific image processing program ImageJ (http://rsb.info.nih.gov/ij/).

Several techniques have been used to improve the signal to noise ratio of the images. The background noise has a 'salt and pepper' characteristic which can be reduced by taking a median of a series of images, or by the 'despeckle' process in ImageJ, which replaces each pixel with the median value in its $3 \times 3$ neighborhood. Some loss of resolution accompanies this process. When 2 or more images of the same experimental conditions are available, a particularly effective noise reduction technique without loss of resolution is to take the minimum, pixel by pixel, of the available images. Figure 3 is a combination of three nominally identical images, as in Fig $2 \mathrm{~d}$.

The background scintillator emission from electrons scattered in the mask is shown in Figure 4 , in which a series of 25 images with the mask at various vertical locations are combined by taking the minimum to remove most of the changing pepper-pot beamlets from the image. The remaining baseline signal is due to the scattering of the bulk of the DARHT electron beam. 


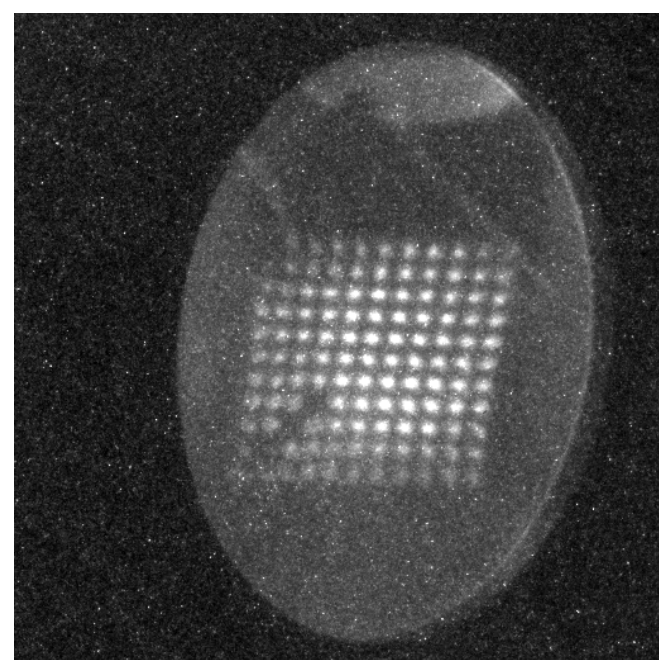

Figure 3. Minimum of three images of the $11 \times 11$ pepper-pot array for identical beam and paddle conditions. Clearly visible is the image of all the holes in the array, as well as the background of scattered electrons in the scintillator.

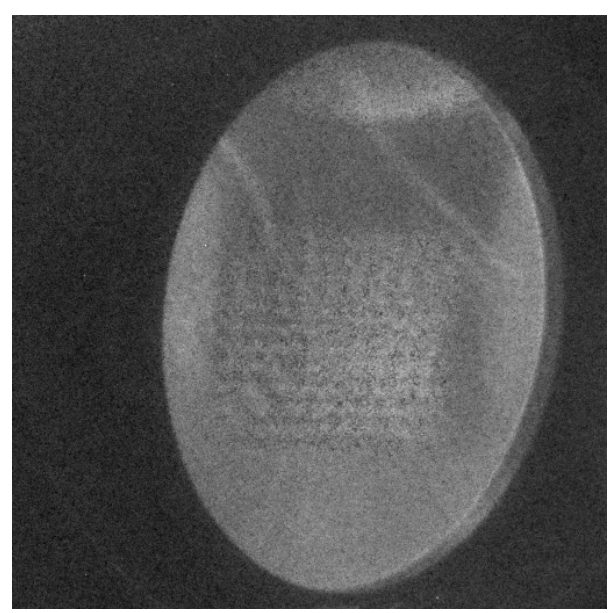

Figure 4. Background light produced by beam electrons striking the scintillator after being scattered by the pepper-pot mask.

The optical beamline or scintillator plate is slightly misaligned causing the image in Fig. 4 to be rotated a few degrees. This rotation can be backed out when analyzing the images. The background light is relatively uniform across the region of the pepper-pot, but there is some structure that may be related to structure in the paddle or in the geometry of the experimental configuration. The scintillator plate appears foreshortened because it is at an angle of 45 degrees with respect to the accelerator axis. The image of the beamlet array is not foreshortened because at 45 degrees the beamlets spread out horizontally an amount equal to the foreshortening of the viewing angle.

The raw images from the PI-MAX camera have a resolution length that is larger than a single pixel. The image of a single point of light is spread out over several pixels due to scattering of light in the process of optical amplification and conversion of the image. Correction of this effect may be done in software by a deconvolution of the image through a point spread function of the camera [Ref. 5] as illustrated in Figure 5. 


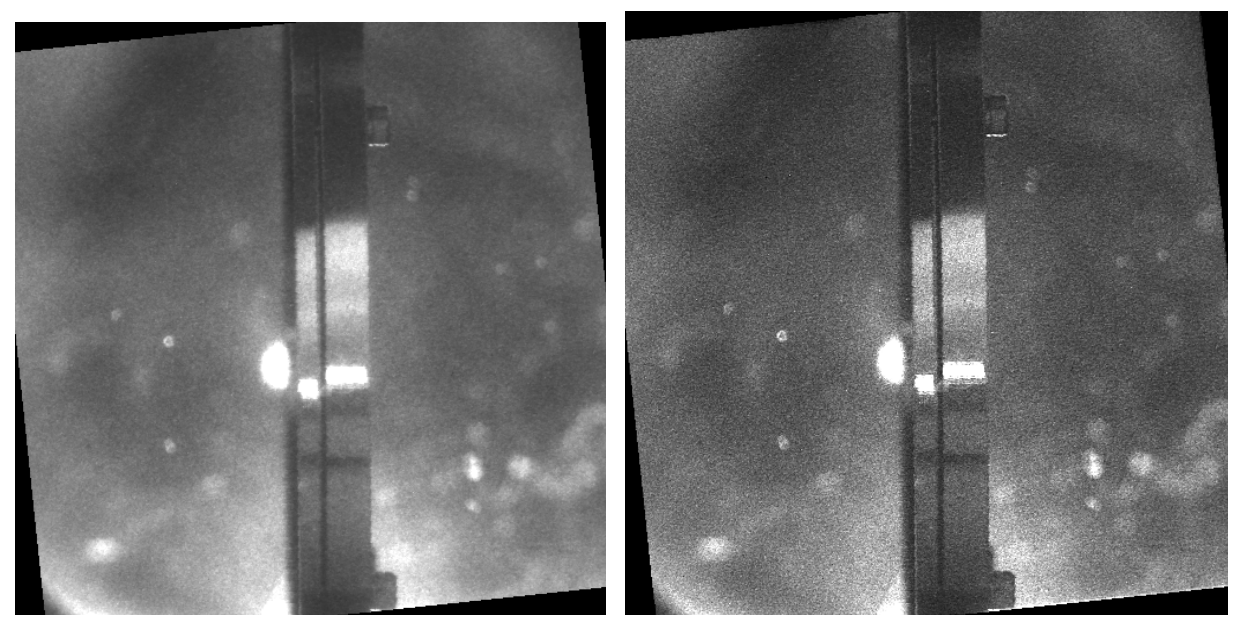

Figure 5. Reference image of a sharp edge using the PI-MAX camera before (left) and after (right) deconvolution of the point spread function. The sharpening is apparent in a visual comparison of the images.

The correction sharpens the image and is necessary in those cases where there are only a few pixels across the image of interest. In the case of the DARHT pepper-pot images, the effect of this correction is relatively minor in determination of the beam emittance. The imaged line is straight, indicating that image distortions, such as pincushion, are not present, at least for a vertical line.
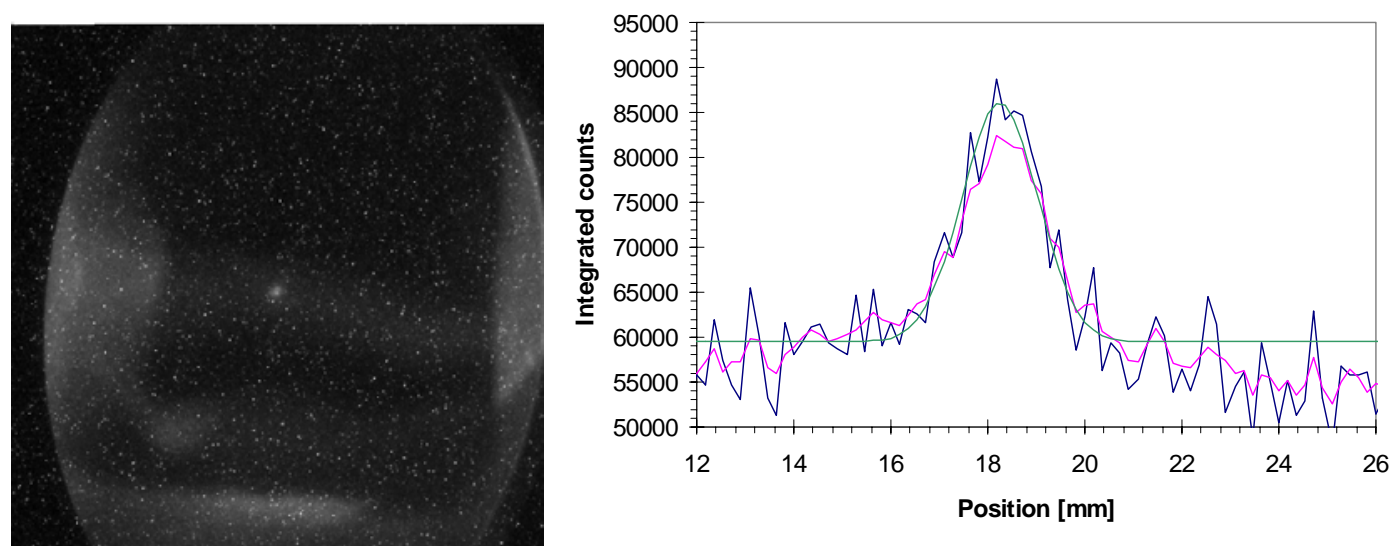

Figure 6. Image of a single pepper-pot hole (shot 2301), and a horizontal line-out of the five combined single-hole images with (blue) and without (magenta) deconvolution. Also shown (green) is a normal distribution with sigma $=0.78 \mathrm{~mm}$. 


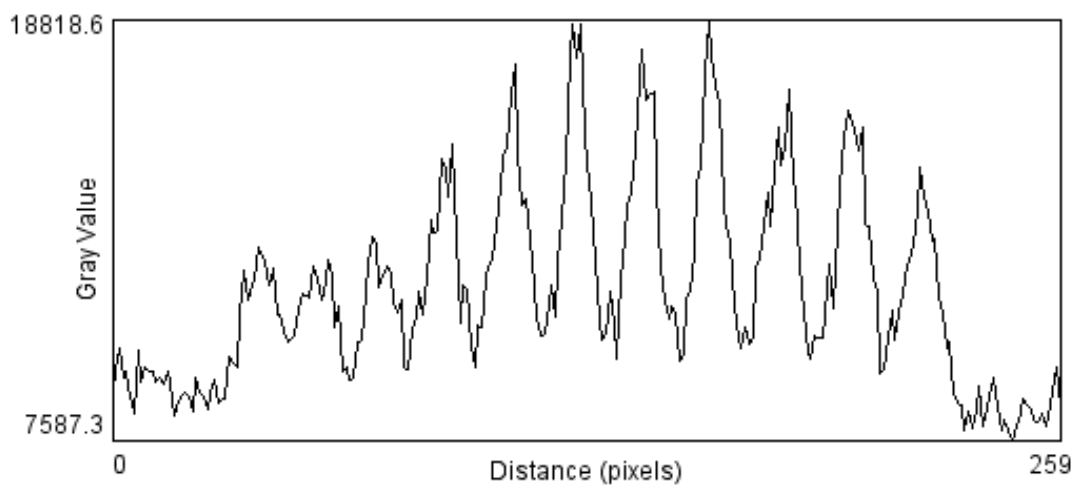

Figure 7. Line integral of a single row of the image of Fig. 3 as a function of horizontal position across the scintillator plate, after deconvolution and rotation by -7 degrees.

\section{Data analysis}

The electron source in the injector is heated by a filament to $\mathrm{T} \approx 1100 \mathrm{C}$. The intrinsic thermal beam emittance at the source of radius $r=8.25 \mathrm{~cm}$ is $\varepsilon_{n}=2 r \sqrt{k T / m c^{2}}=80 \pi \mathrm{mm}$ mrad. Measured beam emittance is much larger because of emittance growth in the injector and accelerator. We define the normalized edge emittance as 4 times the RMS emittance, $\varepsilon_{n}=4 \beta \gamma \varepsilon_{R M S}$, or

$\varepsilon_{n}=4 \beta \gamma \sqrt{\left\langle(x-<x>)^{2}\right\rangle \cdot\left\langle\left(x^{\prime}-<x^{\prime}>\right)^{2}\right\rangle-\left\langle(x-<x>) \cdot\left(x^{\prime}-<x^{\prime}>\right)\right\rangle^{2}}$

in the transverse dimension $\mathrm{x}$ and similarly in $\mathrm{y}$, where $\beta$ and $\gamma$ are the usual relativistic factors, the prime $\left(\mathrm{x}^{\prime}, \mathrm{y}^{\prime}\right)$ corresponds to the spatial derivative $(\mathrm{dx} / \mathrm{dz}, \mathrm{dy} / \mathrm{dz})$ of the transverse dimension, and the bracket notation indicates an average over the particle distribution.

Data for a single pepper-pot hole is shown in Figure 6. Combining the single-hole images greatly improves the signal to noise ratio, but these images were taken with the pepper pot moved around the beam. The beamlet does not strike the same point on the scintillator, especially when the paddle is moved from shot to shot. Great care must be taken to offset the images to ensure that the images line up properly. The line-out of the distribution for the sum of the images across the pinhole is compared with the distribution expected for a normal distribution of particles

$$
f(x)=\frac{1}{\sqrt{2 \pi} \sigma} \exp \left(-\frac{(x-\mu)^{2}}{2 \sigma^{2}}\right)
$$

where $\sigma$ is the standard deviation. The normal distribution fits the data well. The value $\sigma=0.94 \mathrm{~mm}$ fits the raw images and $\sigma=0.78$ fits the deconvolved images. The ratio of the hole size to the measured RMS beam size is 0.53 and 0.64 respectively. This ratio is 
small enough that the correction to the measured beam size and distribution due to the finite size of the hole is less than $10 \%$ [Ref. 6].

Using $2 \sigma$ to calculate emittance corresponds to the 4-RMS emittance, and contains approximately $90 \%$ of the beam. Therefore a rough estimate for the emittance due only to thermal spread of the portion of the beam contained within the region of the pepper-pot array is $2 \sigma * 25 * \beta \gamma \approx 600 \pi \mathrm{mm} \mathrm{mrad}$.

A horizontal lineout for the image of Figure 3 is shown in Figure 7. As expected, there is amount of overlap in the beamlets, indicating a significant beam thermal spread.

Figure 4 shows a series of 15 sequential beam shots with the slit stepped across the beam on a 5 -mm spacing.

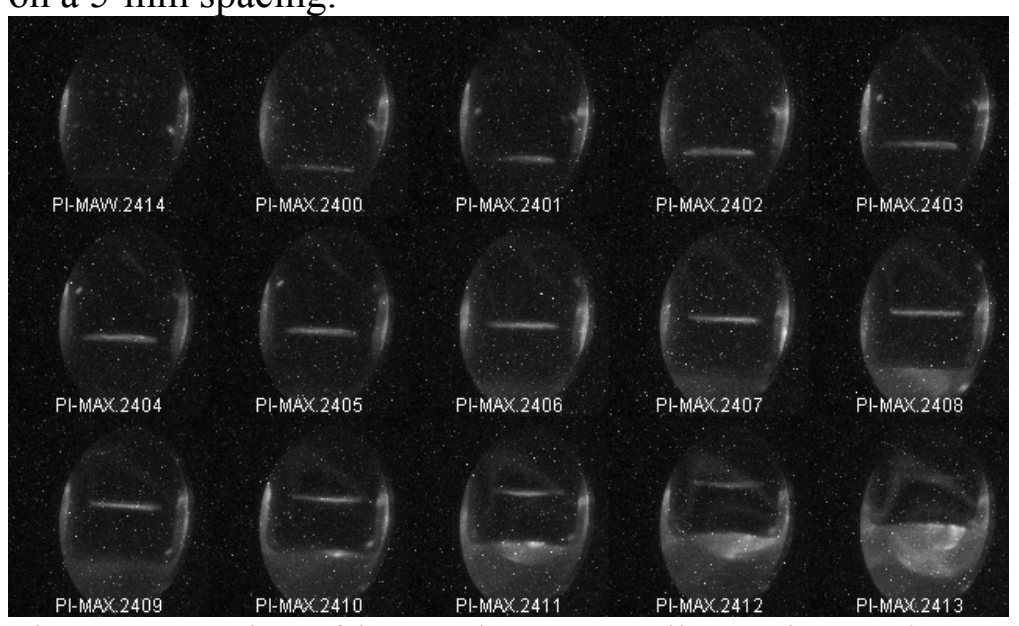

Figure 8. Series of beam shots on a slit can be used to estimate beam phase space and emittance. These images are taken with 5-mm spacing.

Figure 9 shows the cross section of the beam imaged on the quartz plate.

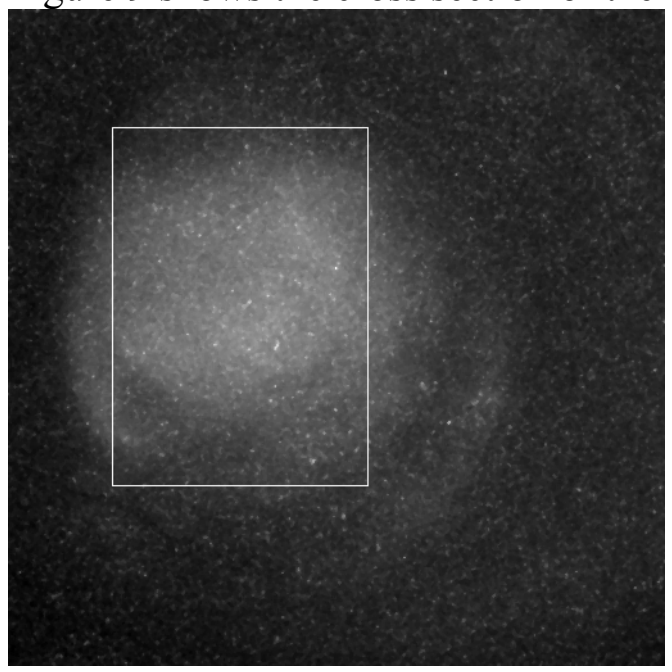

Figure 9. Beam image on quartz plate. Shot \# 2361. 
Also indicated by the box is the 50-mm width of the slit, showing that the tail of the beam distribution is wider than the hole array. Approximately $70 \%$ of the beam is enclosed in the box.

Analysis of the slit scan images was done using the techniques [Ref. 7] developed at LBNL for analysis of heavy ion beams. The graphical summary of results for the scan is shown in Figure 10.

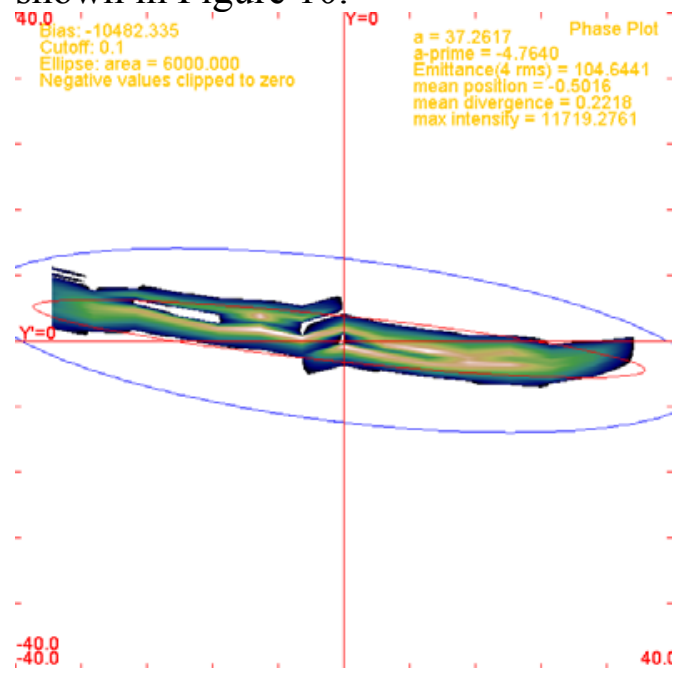

Figure 10. Vertical projection of phase space based on the slit scan of Figure 4.

Thermal emittance is indicated by the vertical spread of the distribution of the data; distortions are deviations from a ellipse. This distribution is dominated by the thermal spread with relatively small overall distortions. The program calculates an edge (4-RMS) emittance of $104 \pi$, or a normalized edge emittance of about $1500 \pi$. The portion of the beam outside the box of Fig. 9 is not included in this estimate, but it will not necessarily add to the final emittance. The blue ellipse in Figure 10 corresponds to the edge of the region included in the analysis (to clip off the unwanted signal at the edges of the paddle) and the red ellipse corresponds to the calculated phase space of the beam. Note that this method of calculating emittance from a series of images is dependent on excellent shotto-shot reproducibility of the beam, which may not be a good assumption. Any variation in the beam position from shot to shot tends toward an overestimate of the beam emittance.

The data shown are for a time window near the beam head, images taken at later times in the beam pulse show similar behavior.

\section{Configurations for injector pepperpot measurements}

The measurements reported here indicate that 1) a phase space measurement at the end of the injector to ascertain source performance is warranted, and 2) the pepperpot technique is applicable to beam measurements at injector exit. 


\section{Appendix A}

We have performed some preliminary calculations to ascertain possible diagnostic locations and magnet tunes for such an experiment. In the first beamline design, we assume that the pepperpot setup is at the exit of the 6-cell injector cell block. The geometry used in the EGUN calculations is shown in Figure 11. Using a total of 7 solenoids from the diode exit, we constructed the magnetic tune to produce a slightly divergent beam of $2.5 \mathrm{~cm}$ radius at the exit of the block.. The magnetic profile together with the resulting beam envelope are shown in Figure 12.

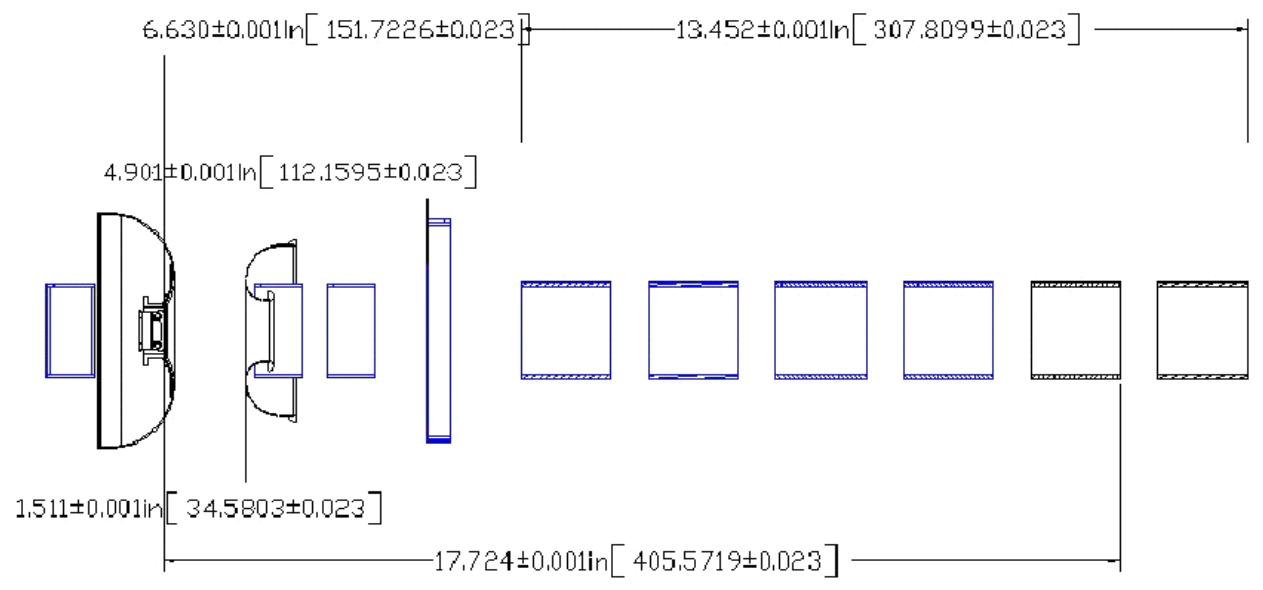

Figure 11. Geometry of diode and injector cell block used the EGUN.

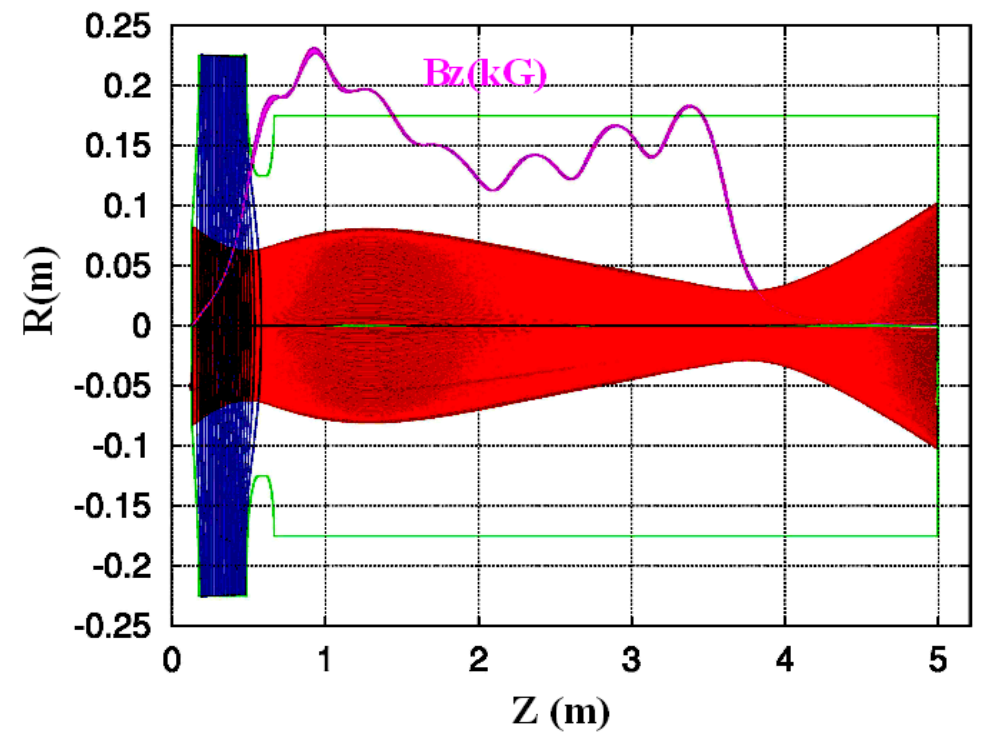

Figure 12. EGUN simulation of $2.5 \mathrm{MeV}, 1.4 \mathrm{KA}$ beam (7 solenoids and bucking coil powered) 
If the pepperpot diagnostic is located at the end of the first injector cell, we can obtain a beam with similar envelope parameters by a tune with somewhat higher magnetic fields. This is shown in Figure 13.

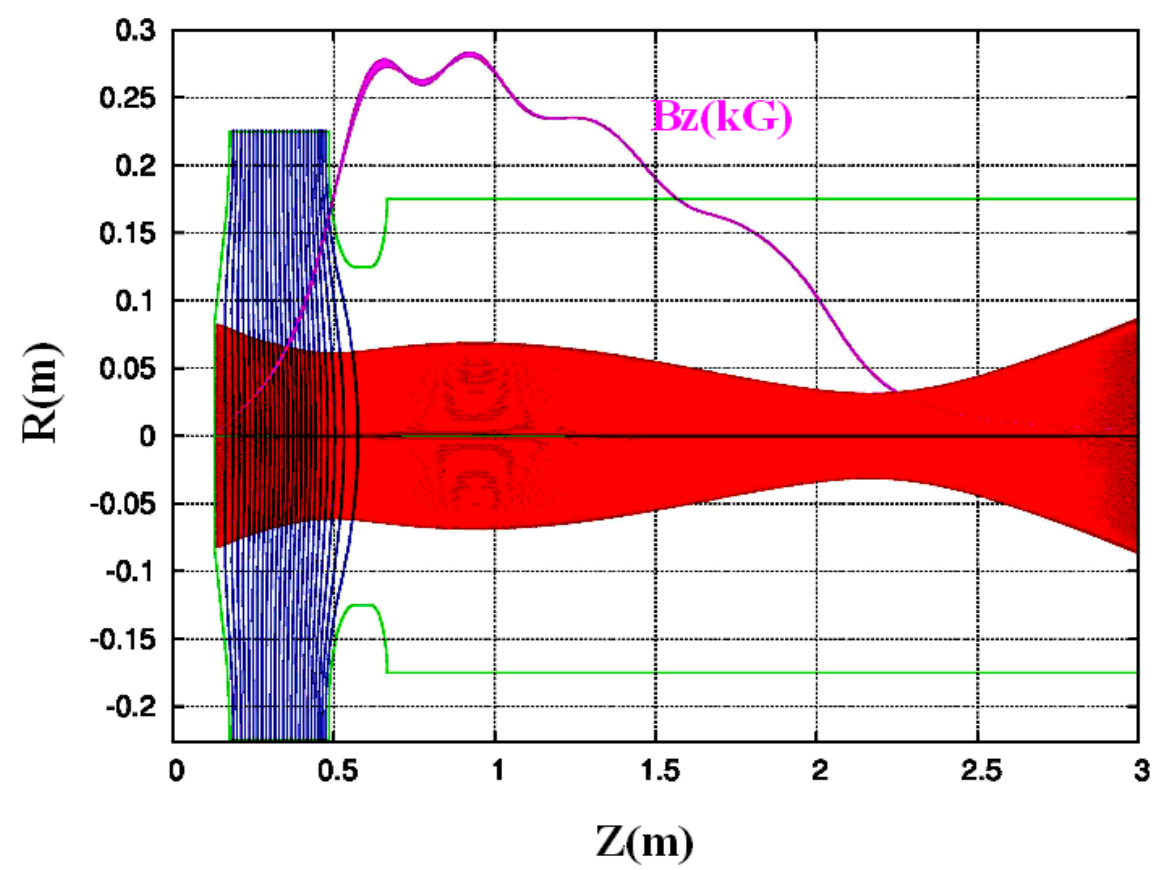

Figure 13. EGUN simulation of $2.5 \mathrm{MeV}, 1.4 \mathrm{KA}$ beam (4 solenoids and bucking coil powered).

\section{Conclusions and future work}

The results of these experiments are

1. The pepper pot experiment has provided useful beam information at the end of the accelerator, and it has promise to be equally useful at the injector location.

2. The beam cross section is roughly symmetric but there are imperfections in the distribution. In addition, there is a large amount of transverse motion in the beam during a beam pulse.

3. The local transverse velocity distribution of the beam implies the normalized emittance from the local thermal spread alone is about 600 pi over a $5 \times 5 \mathrm{~cm}$ region.

4. The normalized emittance of the full beam, including both the local thermal spread and the global distortion of the beam, is approximately 1500 pi based on the assumptions and analysis described above.

Experience from the initial run at this location can be used to improve the diagnostic capability at the Injector location. In particular, the following improvements can be made:

1. An improved camera (with lowered background noise level should be available for the next set of data taking. 
2. The optics should be improved to ensure that the image is zoomed on the region of interest of the pepper pot.

3. The paddles and the mask hole arrays should be widened to block beam from passing around the paddle and to capture a larger portion of the beam.

4. The graphite masks can be made thinner at a lower energy. With a thinner mask the limitation on the beam angle of divergence/convergence is not as severe as in the present experiment.

5. The single slit can be replaced with multiple slits (oriented in horizontal and vertical direction) so that a slit scan can be completed in a single or at most a few beam shots.

\section{References}

1. Thomas J. Fessenden, Measurements of the emittance and current density profile of the beam produced by the ATA Injector, LLNL Report, UCID-19839 (June 27, 1983).

2. Arthur C Paul, Roger Richardson, John Weir, ETA II $6 \mathrm{MeV}$ pepperpot emittance measurement, LLNL Report UCRL-TR-207540 (Oct. 28, 2004).

3. M. Roos, et.al., Review of particle properties, Physics Letters 111B, 33 (1982).

4. F.M. Gicquel, LBNL Engineering Note 10114, 7/11/2005.

5. John C. Russ, The Image Processing Handbook, CRC Press, 2002, p. 317-321.

6. T. Hughes, Mission Research Corp. Report MRC/ABQ-R-1133 (1989).

7. F.M. Bieniosek, et. al., Beam imaging diagnostics for heavy-ion fusion beam experiments, 2003 PAC Conference, Paper WPPB050. 